\title{
An outflow in the Seyfert ESO 362-G18 revealed by Gemini-GMOS/IFU observations *
}

\author{
Pedro K. Humire ${ }^{1}$, Neil M. Nagar ${ }^{1}$, Carolina Finlez ${ }^{1}$, Verónica Firpo ${ }^{2,3}$, Roy Slater ${ }^{4}$, Davide Lena ${ }^{5,6}$, \\ Pamela Soto-Pinto ${ }^{1}$, Dania Muñoz-Vergara ${ }^{1}$, Rogemar A. Riffel ${ }^{7}$, Henrique R. Schmitt ${ }^{8}$, Steven B. Kraemer ${ }^{9}$, \\ Allan Schnorr-Müller ${ }^{10}$, Travis C. Fischer ${ }^{11}$, Andrew Robinson ${ }^{12}$, Thaisa Storchi-Bergmann ${ }^{10}$, \\ Mike Crenshaw ${ }^{13}$, and Martin S. Elvis ${ }^{14}$ \\ ${ }^{1}$ Departamento de Astronomía, Universidad de Concepción, Casilla 160, Concepción, Chile \\ e-mail: phumire@udec.cl \\ 2 Departamento de Física y Astronomía, Universidad de La Serena, La Serena, Chile \\ ${ }^{3}$ Gemini Observatory, Southern Operations centre, La Serena, Chile \\ ${ }^{4}$ Dirección de Formacion General, Facultad de Educación y Cs. Sociales, Universidad Andres Bello, Sede Concepción, autopista \\ Concepción-Talcahuano 7100, Talcahuano, Chile \\ ${ }^{5}$ SRON, Netherlands Institute for Space Research, Sorbonnelaan 2, 3584 CA Utrecht, The Netherlands \\ ${ }^{6}$ Department of Astrophysics/IMAPP, Radboud University, Nijmegen, PO Box 9010, 6500 GL Nijmegen, The Netherlands \\ ${ }^{7}$ Universidade Federal de Santa Maria, Departamento de Física, Centro de Ciencias Naturais e Exatas, 97105-900 Santa Maria, RS, \\ Brazil \\ ${ }^{8}$ Naval Research Laboratory, Washington, DC 20375, USA \\ ${ }^{9}$ Institute for Astrophysics and Computational Sciences, Department of Physics, The Catholic University of America, Washington, \\ DC 20064, USA \\ ${ }^{10}$ Instituto de Física, CP 15015,Universidade Federal do Rio Grande do Sul, 91501-970, Porto Alegre, RS, Brazil \\ ${ }^{11}$ Astrophysics Science Division, Goddard Space Flight centre, Code 665, Greenbelt, MD 20771, USA \\ 12 Department of Physics, Rochester Institute of Technology, 84 Lomb Memorial Drive, Rochester, NY 14623, USA \\ ${ }^{13}$ Department of Physics and Astronomy, Georgia State University, Astronomy Offices, 25 Park Place Suite 605, Atlanta, GA 30303, \\ USA \\ ${ }^{14}$ Harvard-Smithsonian centre for Astrophysics 60 Garden St., ms.6, Cambridge MA 02138 USA
}

Received 28 July 2017 / Accepted 16 February 2018

\begin{abstract}
We present two-dimensional stellar and gaseous kinematics of the inner $0.7 \times 1.2 \mathrm{kpc}^{2}$ of the Seyfert 1.5 galaxy ESO 362-G18, derived from optical (4092-7338 ̊) spectra obtained with the GMOS integral field spectrograph on the Gemini South telescope at a spatial resolution of $\approx 170 \mathrm{pc}$ and spectral resolution of $36 \mathrm{~km} \mathrm{~s}^{-1}$. ESO $362-\mathrm{G} 18$ is a strongly perturbed galaxy of morphological type Sa or S0/a, with a minor merger approaching along the NE direction. Previous studies have shown that the [O III] emission shows a fan-shaped extension of $\approx 10^{\prime \prime}$ to the SE. We detect the [O III] doublet, [N II] and $\mathrm{H} \alpha$ emission lines throughout our field of view.

The stellar kinematics is dominated by circular motions in the galaxy plane, with a kinematic position angle of $\approx 137^{\circ}$ and is centred approximately on the continuum peak. The gas kinematics is also dominated by rotation, with kinematic position angles ranging from $122^{\circ}$ to $139^{\circ}$, projected velocity amplitudes of the order of $100 \mathrm{~km} \mathrm{~s}^{-1}$, and a mean velocity dispersion of $100 \mathrm{~km} \mathrm{~s}^{-1}$. A doubleGaussian fit to the [O III] $\lambda 5007$ and $\mathrm{H} \alpha$ lines, which have the highest signal to noise ratios of the emission lines, reveal two kinematic components: (1) a component at lower radial velocities which we interpret as gas rotating in the galactic disk; and (2) a component with line of sight velocities $100-250 \mathrm{~km} \mathrm{~s}^{-1}$ higher than the systemic velocity, interpreted as originating in the outflowing gas within the AGN ionization cone. We estimate a mass outflow rate of $7.4 \times 10^{-2} M_{\odot} \mathrm{yr}^{-1}$ in the SE ionization cone (this rate doubles if we assume a biconical configuration), and a mass accretion rate on the supermassive black hole (SMBH) of $2.2 \times 10^{-2} M_{\odot} \mathrm{yr}^{-1}$. The total ionized gas mass within $\sim 84 \mathrm{pc}$ of the nucleus is $3.3 \times 10^{5} M_{\odot}$; infall velocities of $\sim 34 \mathrm{~km} \mathrm{~s}^{-1}$ in this gas would be required to feed both the outflow and SMBH accretion.
\end{abstract}

Key words. galaxies: active - galaxies: individual: ESO 362-G18 - galaxies: Seyfert - galaxies: nuclei galaxies: kinematics and dynamics

\section{Introduction}

It is now widely accepted that the intense radiation emitted by an active galactic nucleus (AGN) is due to accretion onto a

\footnotetext{
* The reduced datacube (FITS file) is only available at the CDS via anonymous ftp to cdsarc.u-strasbg.fr (130.79.128.5) or via http://cdsarc.u-strasbg.fr/viz-bin/qcat?]/A+A/614/A94
}

supermassive black hole (SMBH; Lynden-Bell 1969; Begelman et al. 1984) in the mass range $\sim 10^{6}-10^{9} M_{\odot}$. However, the mechanisms responsible for transferring the mass from galactic $(\mathrm{kpc})$ scales down to nuclear scales (sub-parsec) to feed the SMBH are still under debate. This has been the subject of many theoretical and observational studies (Shlosman et al. 1990; Maciejewski 2004a,b; Knapen 2005; Emsellem et al. 2006; Schnorr-Müller et al. 2014a,b, 2017). 
Theoretical studies and simulations have shown that nonaxisymmetric potentials efficiently promote gas inflow towards the inner regions of galaxies (Englmaier \& Shlosman 2004). Close encounters and galactic mergers have been identified as a mechanism capable of driving gas from tens of kiloparsecs down to a few kiloparsecs (Hernquist 1989; Di Matteo et al. 2005). Major mergers are apparently a requirement for triggering the most luminous AGNs (Treister et al. 2012). Simulations by Hopkins \& Quataert (2010) suggest that in gas-rich systems, at scales of 10 to $100 \mathrm{pc}$, inflows are achieved through a system of gravitational instabilities over a wide range of morphologies such as nuclear spirals, bars, rings, barred rings, clumpy disks, and streams.

Indeed, several observations support the hypothesis that large-scale bars channel the gas to the centres of galaxies (Crenshaw et al. 2003). Recent studies have concluded that there is an excess of bars among Seyfert galaxies as compared to nonactive galaxies of about $75 \%$ versus $57 \%$, respectively (Knapen et al. 2000; Laine et al. 2002; Laurikainen et al. 2004). Further, structures such as disks or small-scale nuclear bars and the associated spiral arms are often found in the inner kiloparsec of active galaxies (Erwin \& Sparke 1999; Pogge \& Martini 2002; Laine et al. 2003; Combes et al. 2014). In general, the most common nuclear structures are dusty spirals, estimated to reside in more than half of active and inactive galaxies $(71 \%$ and $61 \%$, respectively; Martini et al. 2003).

Simões Lopes et al. (2007) reported a marked difference in the dust and gas content of early-type active and non-active galaxies: the former always have dusty structures and only $25 \%$ of the latter have such structures. Thus, a reservoir of gas and dust is required for the nuclear activity suggesting that the dusty structures are tracers of feeding channels to the AGN. This fact, along with the enhanced frequency of dusty spirals, supports the hypothesis that nuclear spirals are a mechanism for fueling the $\mathrm{SMBH}$, transporting the gas from kiloparsec scales down to a few tens of parsecs of the nucleus.

Accretion onto the SMBH requires the removal of angular momentum, which can be achieved not only through gravitational torques, but also via outflows or winds (Bridle \& Perley 1984). The most powerful of these outflows are produced by the interaction between the ionized gas and magnetic field (Bisnovatyi-Kogan \& Lovelace 2001) reaching velocities of up to $1000 \mathrm{~km} \mathrm{~s}^{-1}$ (Rupke \& Veilleux 2011; Greene et al. 2012) and outflow rates several times larger than host galaxy star formation rates (SFR; Sturm et al. 2011). Massive AGN-driven outflows have been observed in many AGN, from Seyfert galaxies to quasars at low (Morganti et al. 2007) and high redshifts (Nesvadba et al. 2011), and could dramatically affect the evolution of galaxies due to the large amounts of energy they feed back into the interstellar medium (Di Matteo et al. 2005). At the less powerful end, studies of nearby Seyferts show that compact outflows $\left(\sim 100 \mathrm{pc}\right.$ in extent) with velocities of $\sim 100 \mathrm{~km} \mathrm{~s}^{-1}$ and mass outflow rates of a few solar masses per year are common even in low-luminosity AGNs (e.g. Müller-Sánchez et al. 2011; Davies et al. 2014).

At low outflow velocities, it can be difficult to identify if AGNs or host galaxy starbursts are responsible for the outflow: a cut-off of $500 \mathrm{~km} \mathrm{~s}^{-1}$ is often used (Fabian 2012; Cicone et al. 2014) to differentiate the two. Identifying low-velocity outflows requires relatively high spectral resolutions and two-dimensional spectroscopy (integral-field spectrographs) to disentangle the different velocity components present: from the galactic disk and from outflow(s) and/or inflow(s) (Storchi-Bergmann et al. 2010; Schnorr-Müller et al. 2014b). Moreover, in some special cases these outflows are detected more frequently as redshifted, rather than blueshifted, winds since the light from the ionized regions reaches us preferentially from the receding side of the outflow which, for our line of sight (LOS), is more illuminated by the AGN (e.g. Lena et al. 2015), and the kinematics can often be modelled as a combination of a biconical outflow and a rotating disk coincident with the molecular gas (Müller-Sánchez et al. 2011; Fischer et al. 2013). A better understanding of low-velocity outflows in nearby Seyferts is important to understand the kinematics of Seyfert galaxies at higher redshift, which could share the same model.

Outflows could be very important for the evolution of galaxies because they can be the most efficient way for the interaction between the AGN and its host galaxy, a process called AGN feedback, affecting the interstellar medium and star formation. Several works explored whether this process triggers (positive feedback) or extinguishes (negative feedback) the host galaxy star formation (Fabian 2012; Karouzos et al. 2014). Empirical scaling relations between the masses of the SMBH and the hostgalaxy bulge (e.g. Gültekin et al. 2009), and between the AGN luminosity and the molecular outflow velocity (Sturm et al. 2011) or dynamical mass (Lin et al. 2016), have motivated a more intensive study of outflows.

In this work, we present results obtained from integral field spectroscopy observations of the nuclear region of ESO 362G18 (a.k.a. MCG 05-13-17), a nearby galaxy of morphological type Sa (Malkan et al. 1998, hereafter MGT) or S0/a (RC3; de Vaucouleurs et al. 1991) harbouring a Seyfert 1.5 nucleus (Bennert et al. 2006). ESO 362-G18 has a redshift of 0.012445 and a systemic velocity of $3731 \mathrm{~km} \mathrm{~s}^{-1}$ (Paturel et al. 2003) or $3707 \mathrm{~km} \mathrm{~s}^{-1}$ (Makarov et al. 2014); we consider the former estimate since it represents our data very well. Assuming $H_{0}=73.0 \mathrm{~km} \mathrm{~s}^{-1} \mathrm{Mpc}^{-1}$, this corresponds to a distance of $50.8 \mathrm{Mpc}$ and a linear scale of $246 \mathrm{pc} \operatorname{arcsec}^{-1}$. Previous studies estimated morphological position angles (PA) between $110^{\circ}$ and $160^{\circ}$ (RC3; Fraquelli et al. 2000, and references therein) and a disk inclination (i), ranging from $37^{\circ}$ to $54^{\circ}$ (Fraquelli et al. 2000, RC3, respectively). ESO 362-G18 has been studied in the radio, near-infrared (NIR), optical, UV, and X-ray; its nucleus has typically been classified as Seyfert 1 (MGT; Mulchaey et al. 1996; Rodríguez-Ardila et al. 2000; Fraquelli et al. 2000; Agís-González et al. 2014).

Previous studies indicate that ESO $362-$ G18 is a highly disturbed galaxy (Mulchaey et al. 1996) with a "long faint plume" to the NE (Corwin 1985); this plume is likely an infalling less massive galaxy $10^{\prime \prime}$ to the NE, i.e. a minor merger. The emission-line maps of Mulchaey et al. (1996) revealed strong [O III] emission centred near the continuum peak with a fan-shaped emission of $\sim 10^{\prime \prime}$ in the SE direction, roughly along the host galaxy major axis and coincident with the strongest $\mathrm{H} \alpha$ emission that is more symmetrically distributed about the nucleus. Mulchaey et al. (1996) estimated that the highest excitation gas is located $\sim 7^{\prime \prime}$ SE from the nucleus on one edge of the ionization cone, but Bennert et al. (2006) found that only the central $\pm 3^{\prime \prime}$ show line ratios typical of AGN ionized gas, and confirm the suggestion of Fraquelli et al. (2000) that the ionization parameter is peaked in the nucleus and rapidly decreases within the narrow line region (NLR) based on the increased [OII]/[O III] ratio. Fraquelli et al. (2000) also suggested that the nuclear continuum ionizes the gas in the disk along $\mathrm{PA}=158^{\circ}$, giving rise to the fan-shaped region observed in [O III]. Arcsecond resolution centimeter radio maps of ESO 362-G18 do not show any obvious extensions (Nagar et al. 1999). Bennert et al. (2006) find that the spectra out to $r \sim 11^{\prime \prime} \mathrm{NW}$ and out to $r \sim 6^{\prime \prime} \mathrm{SE}$ have line ratios that fall in 
the regime of H II regions. Indeed, Tsvetanov \& Petrosian (1995) identified $38 \mathrm{H}$ II regions in their ground-based $\mathrm{H} \alpha+[\mathrm{N}$ II] image of ESO 362-G18, distributed in a cloud around the nucleus with distances between $3^{\prime \prime}$ and $18^{\prime \prime}$.

The nuclear optical spectrum is dominated by broad permitted lines and narrow permitted and forbidden lines (Rodríguez-Ardila et al. 2000) and shows a featureless nuclear continuum due to the AGN (Fraquelli et al. 2000), and main stellar features of $\mathrm{Ca}$ II $K, G$ band, $\mathrm{Mg} \mathrm{I}$ b and $\mathrm{Na} \mathrm{I} \mathrm{D,} \mathrm{as}$ well as high order Balmer absorptions lines outside the nucleus. A broad Balmer decrement $\mathrm{H} \alpha_{\text {broad }} / \mathrm{H} \beta_{\text {broad }}$ of 5.7 indicates a slightly higher reddening of the broad line region (BLR) with respect to the central NLR (Bennert et al. 2006).

Near-infrared spectroscopy (Riffel et al. 2006) shows strong, broad $\mathrm{H} \mathrm{I}$, and He I lines with a full width at half maximum (FWHM) of $\approx 4500 \mathrm{~km} \mathrm{~s}^{-1}$ and $\approx 5400 \mathrm{~km} \mathrm{~s}^{-1}$, respectively. Besides, numerous forbidden lines are seen, including highionization lines of [S IX], [Si X], and [Si VI]. The $\mathrm{Br} \gamma$ and $H_{2}$ molecular emission lines are observed as well, although they are intrinsically weak. The NIR continuum emission present stellar absorption features of $\mathrm{Ca}$ II, $\mathrm{CO}$ in $H$ band, and the $2.3 \mu \mathrm{m}$ $\mathrm{CO}$ band heads in $K$ band on top of a steep power-law like the continuum.

The most recent detailed study of this galaxy has been conducted by Agís-González et al. (2014). Their most important result was to detect a large variability in X-ray absorption which they explain as a clumpy, dusty torus lying in a compact region within $\sim 50 \mathrm{r}_{g}$ (probably with $7 \mathrm{r}_{g}, 1 \mathrm{r}_{g}=\mathrm{GM}_{B H} / \mathrm{c}^{2}$ ) from the central black hole. They also estimated an inner accretion disk inclination of $i=53^{\circ} \pm 5^{\circ}$, i.e. aligned with the large-scale galaxy disk $\left(\mathrm{RC} 3 ; 54^{\circ}\right)$.

This paper is organized as follows. Section 2 describes the observations, data processing, and analysis; Sect. 3 presents our results; Sect. 4 discusses the results and presents estimates of the mass outflow and inflow rates; and Sect. 5 presents our conclusions.

\section{Observations, data processing, and analysis software}

The observations were obtained with the integral field unit of the Gemini Multi-Object Spectrograph (GMOS-IFU; Gimeno et al. 2016) at the Gemini South telescope on the night of December 23, 2014 (Gemini project GS-2014B-Q-20). The observations were made in the one-slit mode of GMOS-IFU, in which the science IFU has a field of view (FOV) of $3^{\prime \prime} .5 \times 5^{\prime \prime}$. Two pointings, shifted by $0 . ' 5$ were observed so that the total sky coverage was $4^{\prime \prime} \times 5^{\prime \prime}$, centred on the nucleus. Two exposures of 900 seconds were made at each pointing with a shift in the central wavelength of $50 \AA$ between the two. The seeing at the time of the science observations was 0 ! 7 , as listed in the Gemini observations log, and we confirmed this value by fitting the luminosity profile of the broad line component of $\mathrm{H} \alpha$ (see Sect. 3.5). This corresponds to a linear resolution of $172 \mathrm{pc}$ at the distance of the galaxy. The spectroscopic standard star LTT $1788(V=13.16)$ was observed in a $360 \mathrm{~s}$ exposure $\sim 1 \mathrm{hr}$ before observing ESO 362-G18, under similar atmospheric conditions and with the same instrument set-up.

The selected wavelength range was $4092-7338 \AA$ to cover the $\mathrm{H} \beta \lambda 4861,[\mathrm{O}$ III] $\lambda \lambda 4959,5007, \mathrm{H} \alpha+[\mathrm{N} \mathrm{II}] \lambda \lambda 6548,6583$ and [S II] $\lambda \lambda 6716,6731$ emission lines, observed with the grating GMOS B600-G5323 (set to central wavelength of either $\lambda 5700 \AA$ or $\lambda 5750 \AA$ ) at a spectral resolution of $\mathrm{R} \approx 3534$ at $\lambda 6440 \AA$ corresponding to an instrumental dispersion $\left(\sigma_{\text {inst }}\right)$ of $\approx 36 \mathrm{~km} \mathrm{~s}^{-1}$. Wavelength calibration is expected to be accurate to the order of $8 \mathrm{~km} \mathrm{~s}^{-1}$.

The data reduction was performed using specific tasks developed for GMOS data in the GEMINI.GMOS version 1.13 package and generic tasks in $\operatorname{IRAF}^{1}$. The reduction process (see Lena 2014) comprised bias subtraction, flat-fielding, trimming, wavelength calibration, sky subtraction, relative flux calibration, building of the data cubes at a sampling of $0 .{ }^{\prime} 08 \times 0 .{ }^{\prime} 08$, and finally the alignment and combination of the four data cubes. Owing to signal-to-noise $(\mathrm{S} / \mathrm{N})$ limitations, we used only the overlapping area of the two spatial pointings and also eliminated spaxels at the edge of the IFU. The final science FOV was thus $2^{\prime \prime} 8 \times 4$ ". 8 . Sky subtraction, performed using spectra from the sky IFU. In Fig. 1, we note a residual telluric absorption at $\sim 6870 \AA$ but the [S II] $\lambda \lambda 6716,6731$ emission lines are not affected by these telluric absorptions.

Flux calibration was performed using the spectroscopic standard star LTT $1788(V=13.16)$ for which fluxes are tabulated every $50 \AA$.

In order to measure the stellar kinematics and create an emission-line-only cube, we employed the penalized pixel fitting technique (pPXF; Cappellari \& Emsellem 2004), using single stellar population (SSP) templates derived within the MILES Stellar Library (Sánchez-Blázquez et al. 2006). These templates have a spectral resolution of $2.51 \AA$ (FWHM) or $\sigma_{\text {inst }}$ $\sim 58 \mathrm{~km} \mathrm{~s}^{-1}$ and cover a spectral range of $3525 \AA$ to $7500 \AA$. Although the spectral resolution of the MILES templates is lower than that of our science data $\left(36 \mathrm{~km} \mathrm{~s}^{-1}\right)$, the MILES Stellar Library give better results than, for example, the IndoUS Library $\left(\sigma_{\text {inst }} \approx 30 \mathrm{~km} \mathrm{~s}^{-1}\right.$, Valdes et al. 2004), since the later does not give optimal fits especially within the inner seeing disk. Comparing the pPXF results obtained using the two libraries individually, we observe that the differences in the fits are within the errors for the most part. When running pPXF with the MILES templates we did not convolve either to a lower spectral resolution. This is valid as the intrinsic stellar velocity dispersion in each spaxel is almost always above $60 \mathrm{~km} \mathrm{~s}^{-1}$ (as confirmed when using the Indo-US template library). The resulting stellar velocity dispersion map was corrected for the instrumental resolution of the science data $\left(36 \mathrm{~km} \mathrm{~s}^{-1}\right)$.

Spatial averages (over large and small apertures) of spectra over various regions of the cube were first used to identify the 20 template spectra most used in the fits. These 20 spectra were then used to fit all individual spaxels in the cube. Before running pPXF, we masked spectral regions covering all broad and narrow emission lines; note that the former are present mainly within the inner seeing disk of 0.7 (172 pc). We used a tenth order additive polynomial in pPXF to take away the effects of the continuum shape of the stellar templates, host galaxy, and any AGN powerlaw continuum. The resulting best-fit templates were used to create an emission-line-only spectrum for each spaxel. Examples of this process are shown in Fig. 2.

The centroid velocities, velocity dispersions and the emission-line fluxes of the gas were initially obtained from the emission-line-only cube by fitting a single Gaussian to the $\mathrm{H} \alpha$, $\mathrm{H} \beta,[\mathrm{N} \mathrm{II}],[\mathrm{O} \mathrm{I}],[\mathrm{O} \mathrm{III}],[\mathrm{S} \mathrm{II}] \lambda 6716$ and [S II] $\lambda 6731$ emission

1 IRAF is distributed by the National Optical Astronomy Observatories, which are operated by the Association of Universities for Research in Astronomy, Inc., under cooperative agreement with the National Science Foundation. 

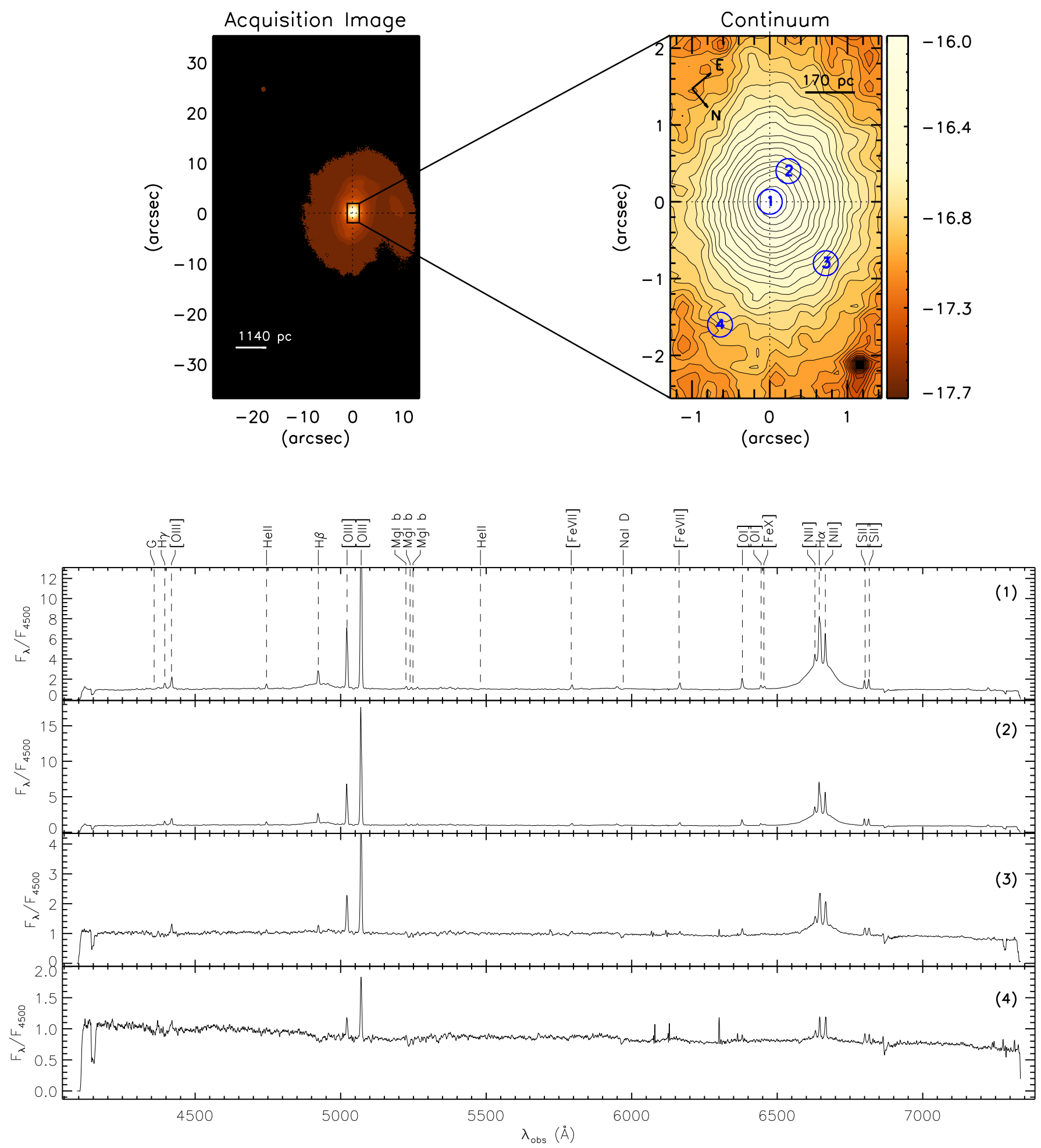

Fig. 1. Top left: acquisition Gemini GMOS image. The rectangle shows the FOV of the IFU observation. Top right: continuum image of ESO 362G18 (see Sect. 3) is shown. Bottom: observed-frame spectra corresponding to the regions are indicated as 1-4 in the IFU image.

lines using FLUXER ${ }^{2}$, which allows us to determine the residual continuum level around the emission lines in an interactive way; this is necessary for the $[\mathrm{S}$ II] lines since they are very close to the broad component of $\mathrm{H} \alpha$. The resulting gaseous velocities are similar to those obtained from the Gas AND Absorption

\footnotetext{
2 Interactive IDL routine written by Christof Iserlohe. http://www. ciserlohe.de/fluxer/fluxer.html
}

Line Fitting code (GANDALF; Sarzi et al. 2006) and PROFIT (Riffel 2010). To obtain the final velocity and dispersion maps we performed a sigma clip of $3 \sigma$ for all radial and velocity dispersion maps except in the nuclear regions of [O III] (because of their high $\mathrm{S} / \mathrm{N})$. We also performed a double-Gaussian fit to the [O III] and $\mathrm{H} \alpha$ emission lines using a series of Python codes. To decide whether the observed line profile is better fit with a single or double Gaussian, we used the corrected Akaike information 

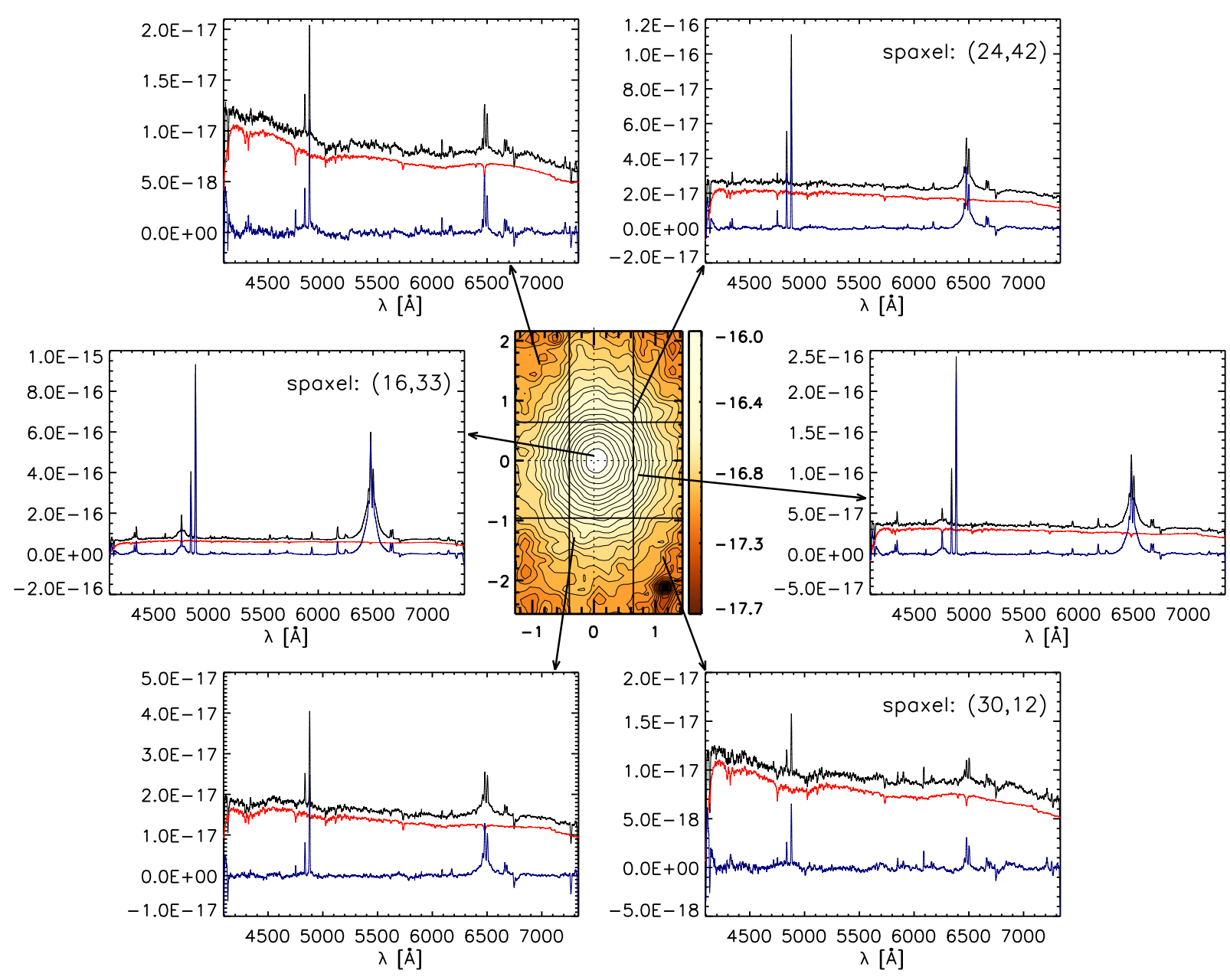

Fig. 2. Fits of the Gemini GMOS spectra of ESO 362-G18 using the MILES Stellar Library (Sánchez-Blázquez et al. 2006) for six spaxels, indicated by the arrows on the continuum image of ESO 362-G18 (the same as Fig. 1 top right panel). The spectra (black lines) and their corresponding fits (red lines) at various locations in the Gemini GMOS field are shown. The vertical and horizontal lines in this continuum image delimit the small apertures where spatial averages of spectra were taken to obtain the spectra most used by pPXF (whose contributions were more important for the best fit to the continuum). The vertical position of the best-fitting spectra obtained with pPXF (Cappellari \& Emsellem 2004) are slightly shifted for legibility; the residual (emission line only) spectra are presented on the bottom (blue lines). Axes units of the continuum image are arcseconds and the $y$ axes of the spectra have units of erg $\mathrm{cm}^{-2} \mathrm{~s}^{-1} \AA^{-1}$.

criterion (Akaike 1974) with the additional caveats that all Gaussian amplitudes are positive. All emission-line velocity dispersion maps were corrected for the instrumental resolution $\left(36 \mathrm{~km} \mathrm{~s}^{-1}\right)$.

The kinematic PAs for both stars and gas were estimated using the code Kinemetry: a generalization of photometry to the higher moments of the LOS velocity distribution (Krajnović et al. 2006). The systemic velocity (hereafter $V_{\text {sys }}$ ) of $3731 \mathrm{~km} \mathrm{~s}^{-1}$ was taken from Paturel et al. (2003).

\section{Results}

The top left panel of Fig. 1 presents the Gemini acquisition image filter $r$ of ESO 362-G18, where the posited minor merger approaching from the NE direction is also clearly seen; the rectangle shows the FOV of the IFU. The top right panel shows the stellar continuum image obtained from our IFU data cube by integrating the flux within a spectral window from $\lambda 5345 \AA$ to $\lambda 5455 \AA$. We assume a nuclear position that coincides with the location of the continuum peak in this image.
In the bottom panel, we present four spectra from the locations indicated as 1 (nucleus), 2 and 3 (intermediate regions) and 4 (boundary region) in the IFU image and extracted within apertures of $0 . ' 2 \times 0$. . 2 . The nuclear spectrum (identified as 1 in Fig. 1) shows broad $\mathrm{H} \alpha$ and $\mathrm{H} \beta$ components, which led to the classification of ESO 362-G18 as a Seyfert 1 galaxy (Fraquelli et al. 2000), and also narrow [O III] $\lambda \lambda 4959,5007 \AA$, [O I] $\lambda \lambda$ $6300,6363 \AA,[\mathrm{N}$ II] $\lambda \lambda 6548,6583 \AA$ and $[\mathrm{S}$ II] $\lambda \lambda 6717,6731 \AA$ emission lines. Large variations in the broad $\mathrm{H} \beta$ emission line of ESO 362-G18 has occasionally led to its classification as a Seyfert 1.5; such variations are not uncommon in Seyfert galaxies.

We created a structure map of ESO 362-G18 (right panel of Fig. 5) by running the IDL routine unsharp_mask.pro on an image obtained with Wide Field Planetary Camera 3 (WFPC3) through the filter F547M aboard the Hubble Space Telescope (HST; Program ID 13816). Inspection of the dust structure shows signs of spiral arms together with stronger obscuration to the SW than to the NE. We thus conclude that the SW is the near side of the galaxy. This is also consistent with flux asymmetries (Sect. 3.1) and a trailing spiral pattern (Sect. 3.2). 


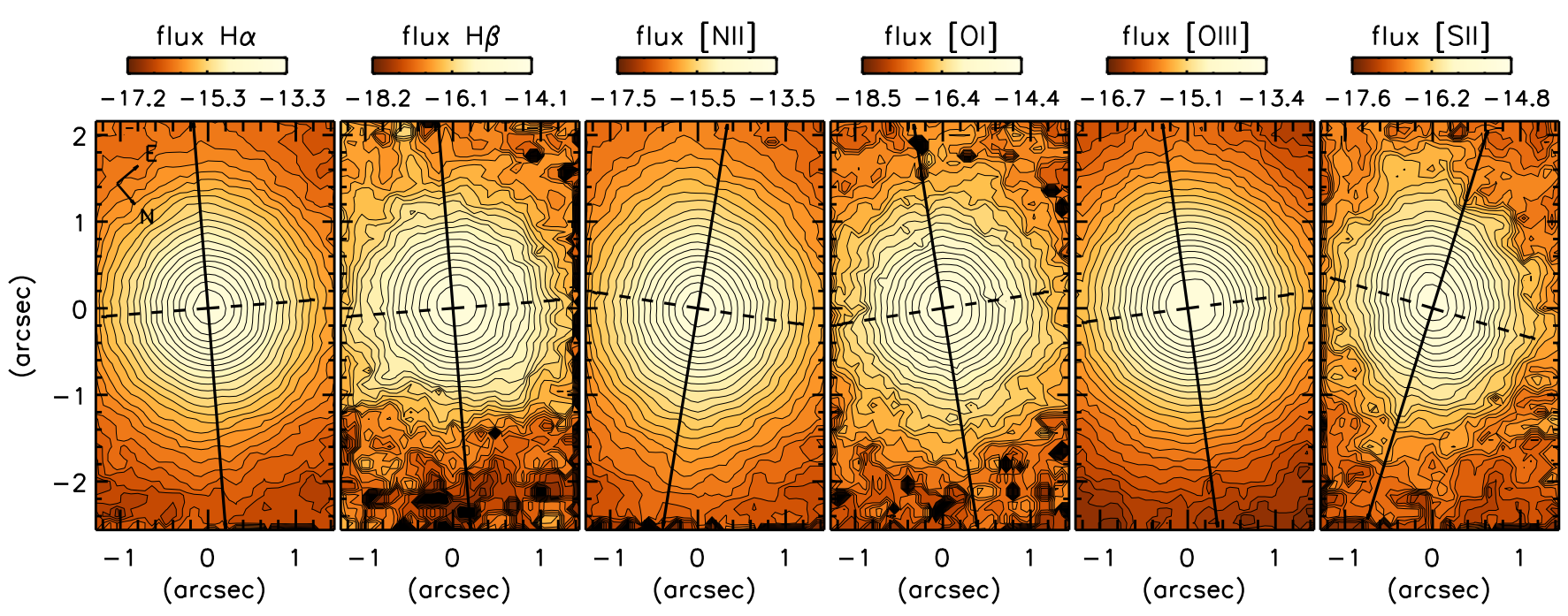

Fig. 3. Maps of the $\mathrm{H} \alpha, \mathrm{H} \beta$, [N II], [O I], [O III] and [S II] integrated fluxes in logarithmic scale ( $\mathrm{erg} \mathrm{cm}^{-2} \mathrm{~s}^{-1}$ per pixel) following the colour bar above each panel. Axes are in arcseconds with respect to the nuclear continuum peak. The solid (dashed) lines indicate the major (minor) axis of the kinematics of the respective line, and the intersection between the lines indicate the continuum peak.
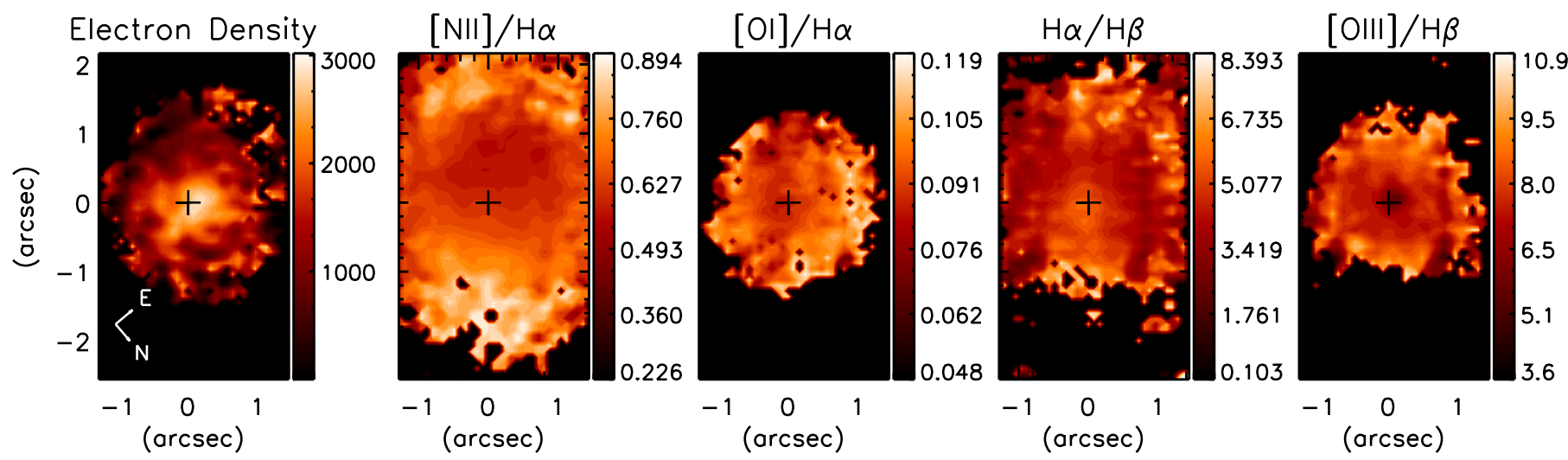

Fig. 4. Maps of the estimated electron density $\left(\mathrm{cm}^{-3}\right)$ distribution and the line ratios $[\mathrm{N}$ II $] / \mathrm{H} \alpha$, [O I $] / \mathrm{H} \alpha, \mathrm{H} \alpha / \mathrm{H} \beta$, and $[\mathrm{O}$ III $] / \mathrm{H} \beta$. The cross represents the continuum peak and each panel follows the colour bar shown on its right.

\subsection{Morphology and excitation of the emitting gas}

In Fig. 3. we present the flux distributions derived from single Gaussian fits to the $\mathrm{H} \alpha, \mathrm{H} \beta,[\mathrm{N} I \mathrm{I}] \lambda 6583 \AA$, [O I] $\lambda 6300 \AA$, [O III] $\lambda 5007 \AA$ and [S II] $\lambda 6716 \AA$ emission lines. The flux distributions show a relatively smooth and symmetric pattern for all the emission lines, slightly elongated along the kinematic major axis (see Figs. 6 and 7), as expected given the inclination. The highest fluxes are within the inner $1^{\prime \prime}(246 \mathrm{pc})$. If the best-fitting two-dimensional Gaussian is subtracted from these flux distributions, the greatest asymmetries are found in the SW, implying a stronger presence of dust here. This supports our previous interpretation of the SW as the near side of the galaxy.

Maps of the estimated electron density and the $[\mathrm{N} I \mathrm{II}] / \mathrm{H} \alpha$, $[\mathrm{O} \mathrm{I}] / \mathrm{H} \alpha, \mathrm{H} \alpha / \mathrm{H} \beta,[\mathrm{O} \mathrm{III}] / \mathrm{H} \beta$ line ratios are presented in Fig. 4. The electron density was obtained from the [S II] $\lambda \lambda 6716 / 6731 \AA$ line ratio assuming an electronic temperature of $10000 \mathrm{~K}$ (Osterbrock \& Ferland 2006). The electron density reaches a peak value of $\sim 2900 \mathrm{~cm}^{-3}$ at the nucleus, decreasing to $1000 \mathrm{~cm}^{-3}$ at $1^{\prime \prime}$ and $800 \mathrm{~cm}^{-3}$ at $1.5^{\prime \prime}$ from the nucleus. These values are in agreement with those obtained by Bennert et al.
(2006) who estimated values from $1000 \mathrm{~cm}^{-3}$ up to $2500 \mathrm{~cm}^{-3}$ in that region.

The $[\mathrm{N} \mathrm{II}] / \mathrm{H} \alpha$ line ratio shows values of $0.55-0.78$ within the inner $1^{\prime \prime}$ and reach its highest values of close to 0.9 in a nuclear-centred ring of radius 1.5." The $[\mathrm{O} \mathrm{III}] / \mathrm{H} \beta$ ratio varies between 5.6 and 9 in the inner $0.5^{\prime \prime}$ and has a depression in the nucleus and increasing to 11 at 1. ." Taking into account both the line ratios, the values can be considered typical of Seyfert galaxies (Cid Fernandes et al. 2010).

\subsection{Stellar kinematics}

The stellar velocity $\left(\mathrm{V}_{\star}\right)$ field, obtained from $\mathrm{pPXF}$, is shown in the left panel of Fig. 5. This field displays a rotation pattern reaching amplitudes of $\approx 75 \mathrm{~km} \mathrm{~s}^{-1}$ within our FOV; the line of nodes are orientated approximately along the NW-SE direction with the SE side approaching and the NW side receding. With our adopted orientation, this implies trailing spiral arms as expected. The stellar velocity dispersion (fourth panel of Fig. 5) reaches values of $120 \mathrm{~km} \mathrm{~s}^{-1}$ at the nucleus, staying up to $100 \mathrm{~km} \mathrm{~s}^{-1}$ to the NW and decreasing to $75 \mathrm{~km} \mathrm{~s}^{-1}$ to the 


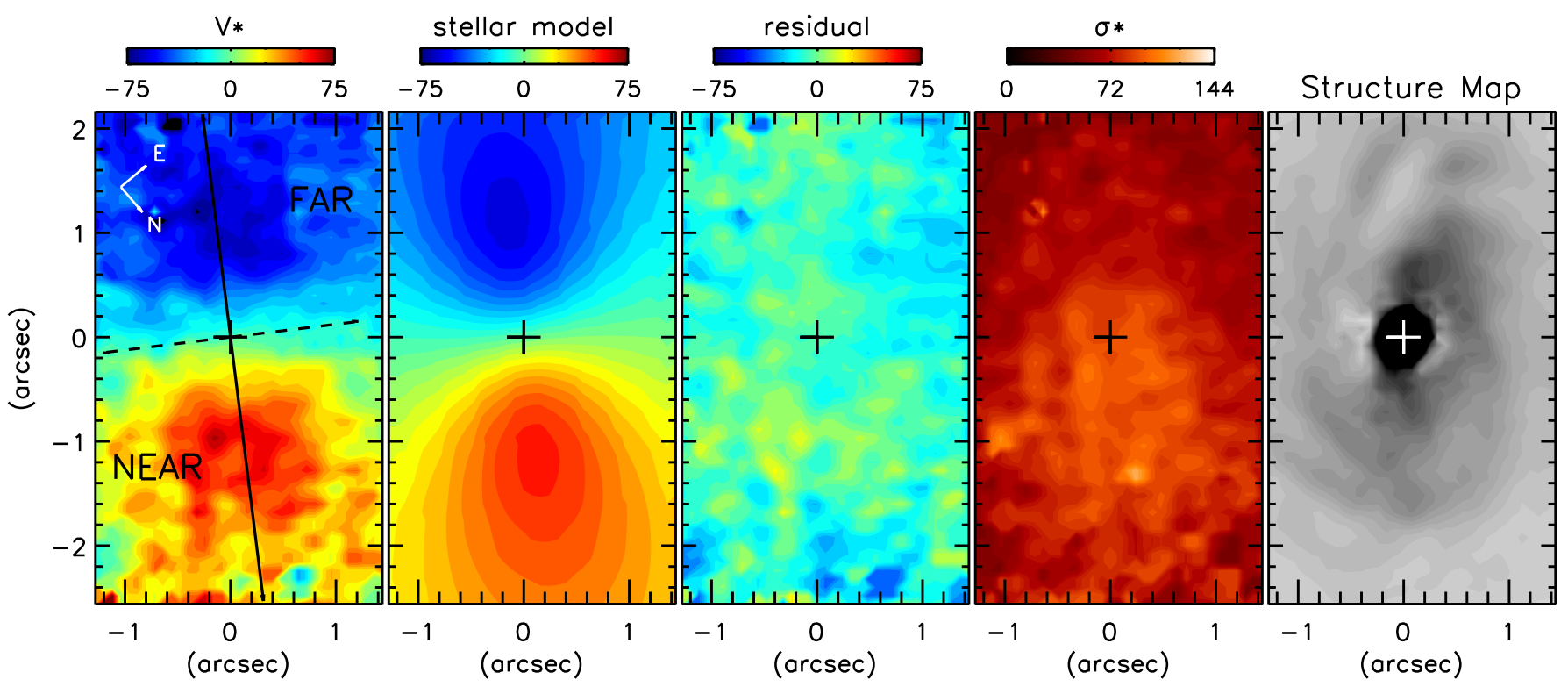

Fig. 5. Stellar velocity field, stellar rotation model, residual velocity field (observed - model), stellar velocity dispersion (all in units of km $\mathrm{s}^{-1}$ following the colour bars above panels), and the structure map (the bright nucleus masked to show details better at fainter fluxes) for ESO $362-$-G18. In the left panel the solid (dashed) black line indicates the position of the kinematic major (minor) axis. The cross denotes the continuum peak in all panels.
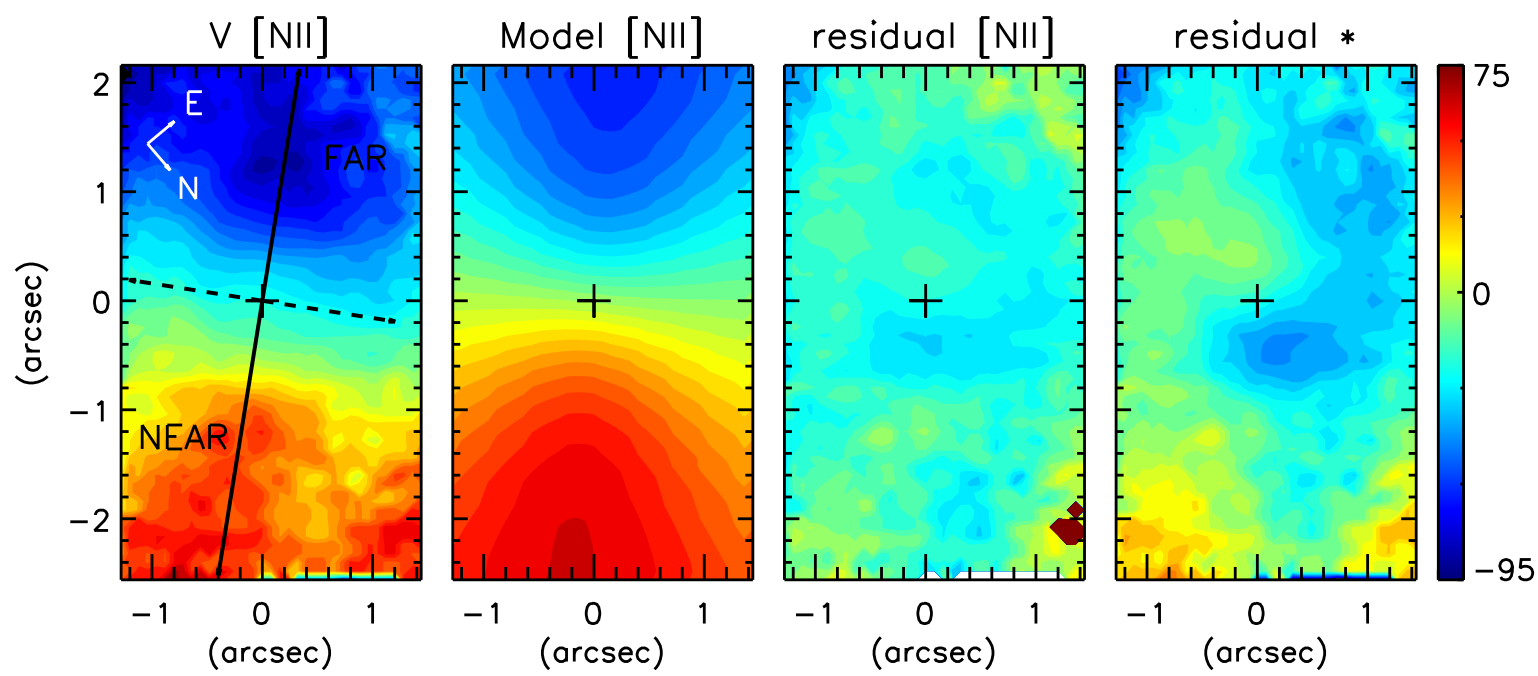

Fig. 6. Observed [N II] velocity field, [N II] rotation model, residual velocity field (observed - [N II] model), and residual velocity field (observed - stellar model). The solid (dashed) black line indicates the position of the kinematic major (minor) axis. The cross represents the continuum peak. All panels follow the colour bar $\left(\mathrm{km} \mathrm{s}^{-1}\right)$ shown on the right.

SE and towards the edges of the FOV. Median radial velocity errors reported by pPXF are $32.6 \mathrm{~km} \mathrm{~s}^{-1}$ in the inner 0.75 and $33.2 \mathrm{~km} \mathrm{~s}^{-1}$ in the inner $1^{\prime \prime} 25$. Median errors in the velocity dispersion (also from pPXF) are $30.0 \mathrm{~km} \mathrm{~s}^{-1}$ in the inner 0.75 , and $40.2 \mathrm{~km} \mathrm{~s}^{-1}$ in the inner $1^{\prime \prime} .25$.

We employed Kinemetry (Krajnović et al. 2006), in which the kinematic centre is fixed to the continuum peak, to obtain the PA of the stellar kinematics at various radii from the nucleus. The resulting values range from $130^{\circ}$ to $139^{\circ}$, thus we chose the median value of $137^{\circ}$ as the "global kinematic" PA, as suggested by Krajnović et al. (2006, Appendix C). This value is consistent with the morphological major axes from the literature of between $110^{\circ}$ and $160^{\circ}$ (RC3, Fraquelli et al. 2000, and references therein).
We model the stellar velocity field by assuming circular orbits in a plane and a spherical potential (Bertola et al. 1991), where the observed radial velocity at a position $(R, \psi)$ in the plane of the sky is given by

$$
V=V_{s}+\frac{A R \cos \left(\psi-\psi_{0}\right)}{\left[R^{2}\left[\sin ^{2}\left(\psi-\psi_{0}\right)+\cos ^{2} \theta \cos ^{2}\left(\psi-\psi_{0}\right)\right]+c^{2} \cos ^{2} \theta\right]^{p / 2}},
$$

where $\theta$ is the inclination of the disk (with $\theta=0$ for a face-on disk), $\psi_{0}$ is the PA of the line of nodes measured with respect to the $x$-axis within the field shown, $V_{\mathrm{s}}$ is the systemic velocity, $R$ is the radius and $A, c$, and $p$ are parameters of the model. We assumed the kinematical centre to be cospatial with the peak of the continuum emission, a PA of $137^{\circ}$ as derived via Kinemetry above, a disk inclination of $37^{\circ}$, obtained from the apparent axial 

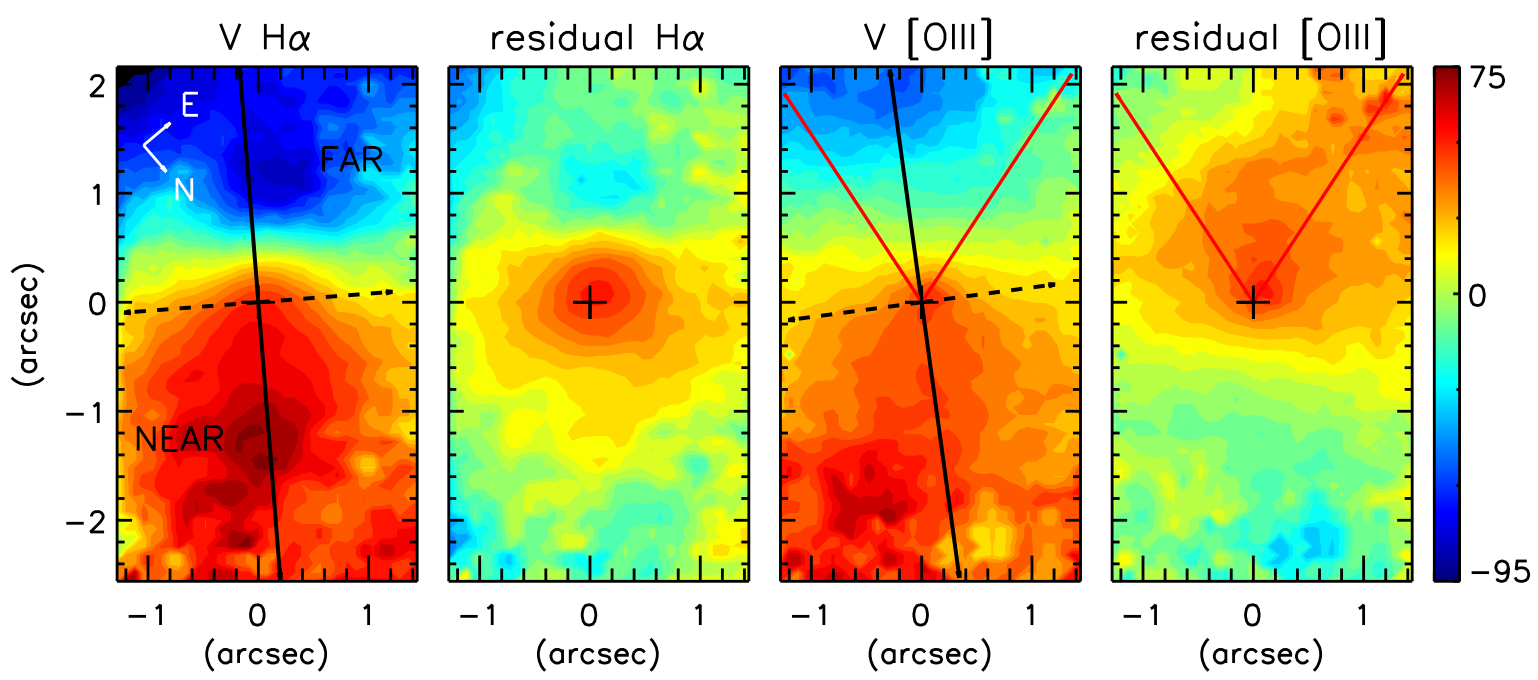

Fig. 7. Emission line gas velocity fields and residual velocity fields (observed - [N II] model) for $\mathrm{H} \alpha$ and [O III]. Panels 1 and 3 show the gaseous centroid velocities $\left(\mathrm{km} \mathrm{s}^{-1}\right)$ obtained from a single Gaussian fit. The cross denotes the location of the stellar continuum peak, and the solid and dashed lines delineate the major and minor kinematic axes as derived using kinemetry (Sect. 3.3). For the [O III] velocity map (and residual velocity field) the red lines denote the bounds of the ionization cone; we use an opening angle of $70^{\circ}$ in agreement with the value $\left(\geq 60^{\circ}\right)$ proposed by Fraquelli et al. (2000). All panels follow the colour bar $\left(\mathrm{km} \mathrm{s}^{-1}\right)$ shown on the right.

ratio (Winkler 1997; Fraquelli et al. 2000) under the assumption of a thin disk geometry, and an initial guess of $3731 \mathrm{~km} \mathrm{~s}^{-1}$ for $V_{\text {sys }}$ (Paturel et al. 2003). We used Levenberg-Marquardt least-squares algorithm to fit the rotation model to the velocity map. The resulting parameters $A, c$ and $p$, are $203 \mathrm{~km} \mathrm{~s}^{-1}, 1^{\prime \prime} .09$ and 1.93 , respectively. The fitted $V_{\text {sys }}$ corrected to the heliocentric reference frame is very similar to our initial guess, so we continue to use the latter. The model stellar velocity field and velocity residuals are shown in Fig. 5.

\subsection{Gas kinematics}

The velocity fields of all strongly detected emission lines, that is $\mathrm{H} \alpha, \mathrm{H} \beta,[\mathrm{N}$ II] $26583 \AA$, [O I] $\lambda 6300 \AA$, [O III] $\lambda 5007 \AA$ and [S II] $\lambda 6716 \AA$, show clear signatures of rotation; the projected peak rotation velocities range from $66 \mathrm{~km} \mathrm{~s}^{-1}$ up to $82 \mathrm{~km} \mathrm{~s}^{-1}$, although non-rotation signatures and offset kinematical centres are also present in most lines. The velocity maps from a single Gaussian fit of the [N II] $\lambda 6583 \AA$, H $\alpha$, and [O III] $\lambda 5007 \AA$ emission lines are shown in the left column of Fig. 6 and first and third panels of Fig. 7.

Inspecting the velocity maps of these emission lines, we find an offset of $\approx 0 . ' 5$ (128pc) between the continuum peak and kinematic centre for $\mathrm{H} \alpha, \mathrm{H} \beta$, [O I] , and [O III], while no significant offset is present in $[\mathrm{N}$ II $] \lambda 6583$ or $[\mathrm{S}$ II $] \lambda 6716$. To more clearly visualize these offsets, we plot the rotation curves of the stars and the stronger emission lines along their respective kinematic PAs in Fig. 8. The offsets cause an apparent asymmetry in the velocity fields within our FOV, reaching greater blueshifts than redshifts in the majority of cases. However, comparing this feature with previous long-slit spectroscopy of the inner $10^{\prime \prime}$ and 30" (Bennert et al. 2006; Fraquelli et al. 2000, respectively), we can infer that this asymmetry is exclusively due to the offset in the kinematic centre.

We used Kinemetry to fit the velocity maps of all emission lines. Given that the rotation curves of the emission lines are offset from that of the stars and each other we obtained reasonable results from Kinemetry only if the kinematic centre was set to a position 0.5 to the $\mathrm{SE}$ of the continuum peak for $\mathrm{H} \alpha$,
$\mathrm{H} \beta$, [O I] $\lambda 6300 \AA$, and [O III] $\lambda 5007 \AA$; for [N II] $\lambda 6583 \AA$ and [S II] $\lambda 6716 \AA$, setting the kinematic centre to the continuum peak gave meaningful results.

Fitting the individual emission line velocity fields with Kinemetry resulted in global kinematic PAs ranging between $121^{\circ}$ and $139^{\circ}$. For each given emission line, the radial variations of the PA do not exceed $20^{\circ}$, and all emission line global kinematic PAs are in rough agreement (within $16^{\circ}$ ) with the stellar kinematic PA except for [S II] for which the difference is $24^{\circ}$. While we expected similar kinematics in $\mathrm{H} \alpha$ and $\mathrm{H} \beta$, Kinemetry gives global PAs of $139^{\circ}$ for $\mathrm{H} \alpha$ and $130^{\circ}$ for $\mathrm{H} \beta$, and indeed at most radii the fitted PA for $\mathrm{H} \alpha$ is $\sim 9^{\circ}$ larger than that of $\mathrm{H} \beta$. We thus use a global PA of $134.5^{\circ}$ for both $\mathrm{H} \alpha$ and $\mathrm{H} \beta$.

Since the $[\mathrm{N}$ II] emission line is both strong and without a significant kinematic offset from the stellar continuum peak, we fit a gas-kinematic Bertola model to its velocity field, following the procedure outlined in Sect. 3.2. Once more we fix the disk inclination to $37^{\circ}, V_{\text {sys }}$ to $3731 \mathrm{~km} \mathrm{~s}^{-1}$, and a kinematic centre cospatial with the stellar continuum peak. The resulting values are $125 \mathrm{~km} \mathrm{~s}^{-1}, 1^{\prime \prime} .06,1.04$, and $125^{\circ}$ for $\mathrm{A}$, $\mathrm{c}, \mathrm{p}$ and the PA, respectively. This gas-kinematics model is shown in the second column of Fig. 6. The third and fourth columns of Fig. 6 show the residual velocity fields of the emission line gas after subtraction of gas-kinematics model and the stellar velocity model, respectively. For all lines except [N II] and $[\mathrm{S}$ II] we see the above-mentioned excess redshift SE of the nucleus.

Maps of the velocity dispersion (henceforth referred to as $\sigma$ before the name of the corresponding emission line) of the corresponding emission lines, derived with a single Gaussian fit, are shown in Fig. 9. The uncertainty here is $\sim 36 \mathrm{~km} \mathrm{~s}^{-1}$ (instrumental dispersion, $\sigma_{\text {inst }}$ ). The highest nuclear dispersions, $\gtrsim 200 \mathrm{~km} \mathrm{~s}^{-1}$, are seen in $\sigma_{H \alpha}$ and $\sigma_{[\mathrm{N} \mathrm{II}]}$; as we leave the nucleus, their dispersions decrease faster along the kinematic major axis than along the kinematic minor axis. On the other hand, $\sigma_{\text {[O III] }}$ is predominantly homogeneous in the non-nuclear regions with its nuclear value rapidly increasing to $170 \mathrm{~km} \mathrm{~s}^{-1}$. In the following sections, we interpret this large nuclear dispersion as due to the presence of a new offset velocity component that is most 


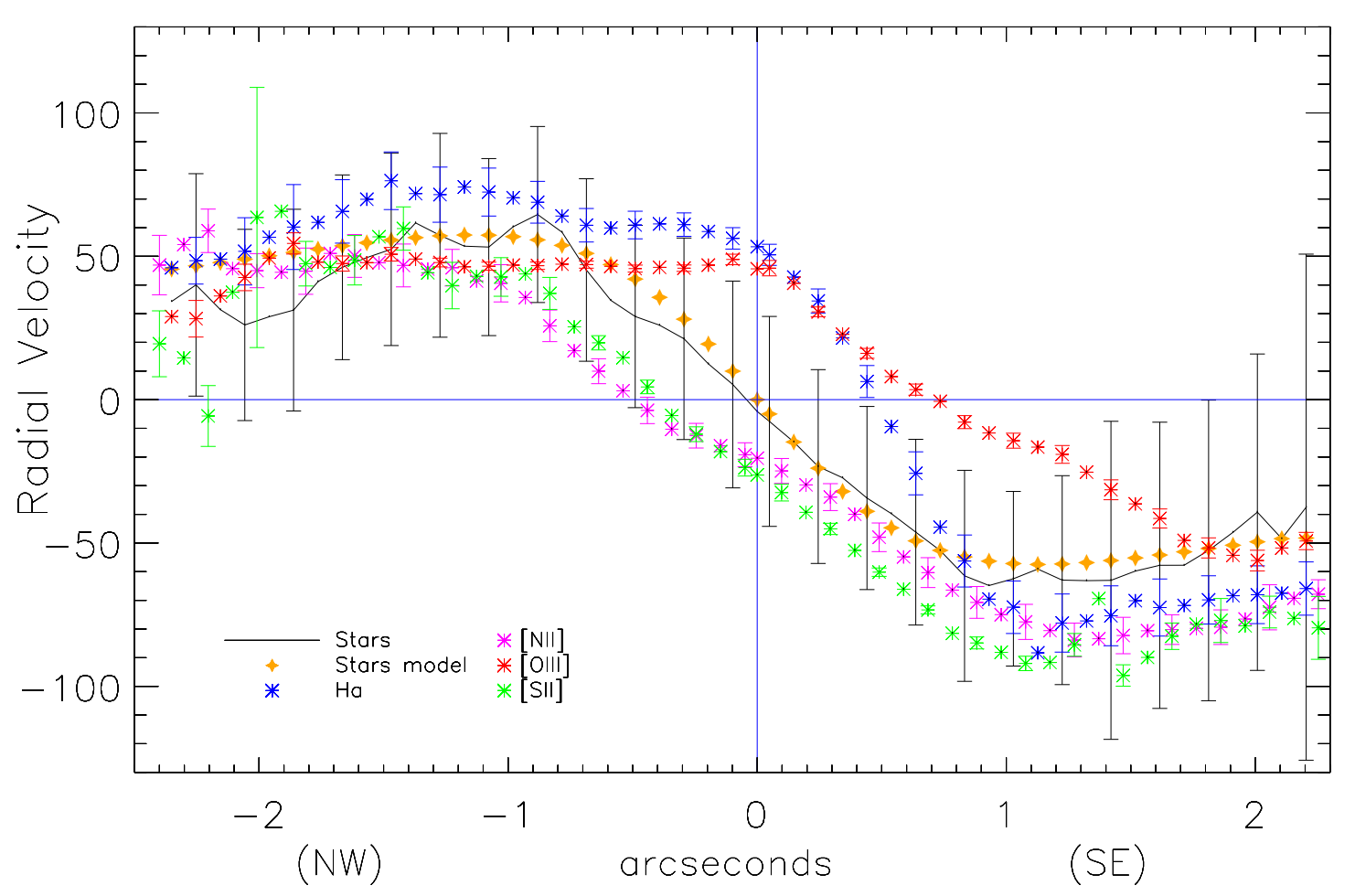

Fig. 8. Radial velocity (rotation curves) curves of the emission lines along with the Bertola model (Stars model) for the stellar kinematics, following the legend in the bottom left. The PAs along which these velocities were taken are those derived by Kinemetry (Sect. 3.3) for each emission line: $135^{\circ}$ for $\mathrm{H} \alpha, 121^{\circ}$ for $[\mathrm{N} \mathrm{II}], 137^{\circ}$ for [O III] and $113^{\circ}$ for [S II]. The zero velocity (horizontal line) corresponds to a (heliocentric) velocity recession of $3731 \mathrm{~km} \mathrm{~s}^{-1}$. Error bars in the corresponding colour indicate the velocity errors determined by pPXF (stars) or Fluxer (Gaussian fitting to emission lines). The vertical line denotes the position of the continuum peak.

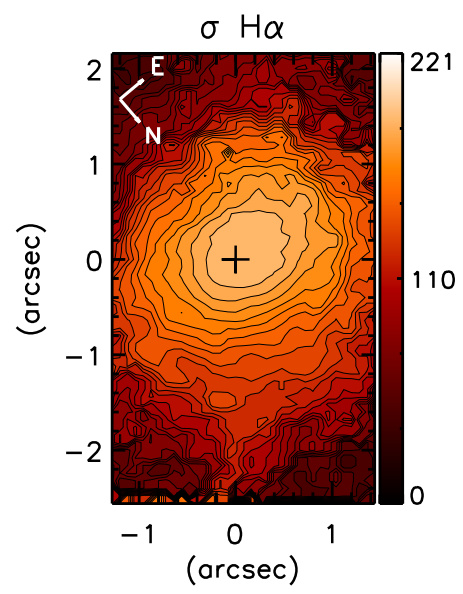

prominent in the nuclear region causing blended profiles and thus non-reliable fits to a single Gaussian. The dispersion of the $\mathrm{H} \beta$ line is similar to that of $\mathrm{H} \alpha$, while both $\sigma_{[\mathrm{OI}]}$ and $\sigma_{[\mathrm{S} \mathrm{II}]}$ do not present centrally peaked distributions.

Radial velocity errors were taken directly from FLUXER (Sect. 2). For $\mathrm{H} \alpha$, [O III] and [N II] emission lines, these errors vary between 1 and $24 \mathrm{~km} \mathrm{~s}^{-1}$ in the inner $1^{\prime \prime} 25$. As the remaining emission lines observed are not present throughout our FOV, we obtained the errors within the inner 0 . 75 , where they vary in the range between 0.8 and $27 \mathrm{~km} \mathrm{~s}^{-1}$. Errors in the velocity dispersion (also from FLUXER) for $\mathrm{H} \alpha$, [O III] and [N II] emission lines vary between 0.7 and $16 \mathrm{~km} \mathrm{~s}^{-1}$ in the inner 1 '.'25, and between 1.6 and $29 \mathrm{~km} \mathrm{~s}^{-1}$ in the inner 0.75 for the remaining emission lines.

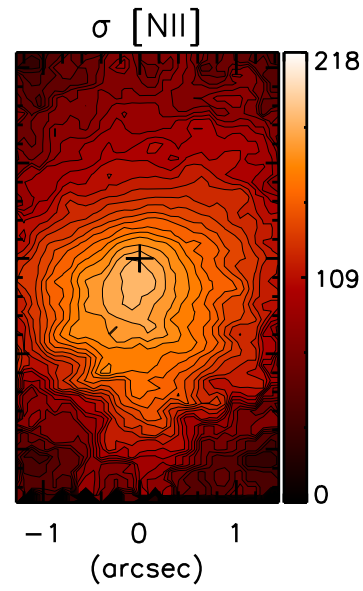

Fig. 9. Velocity dispersions $\left(\mathrm{km} \mathrm{s}^{-1}\right)$ for $\mathrm{H} \alpha$, [O III], and [N II] emission lines. Each colour bar $\left(\mathrm{km} \mathrm{s}^{-1}\right)$ is centred on the average velocity dispersion of the respective line. Crosses denote the continuum peak.

\subsection{Position-velocity diagrams}

To better constrain the emission-line kinematics we built position-velocity (PV) diagrams (Fig. 10) for the three strongest emission lines, [O III], $\mathrm{H} \alpha$, and [N II]. We centred these PV diagrams on the continuum peak and along PA $130^{\circ}$ since this is the kinematic major axis found in the single Gaussian fit. The pseudo slit is 0.8 wide. The velocity prediction from the single Gaussian fit is superposed for an easy direct comparison.

While the PV diagram of [N II] shows a good agreement with the single Gaussian fit, the diagrams of both [O III] and $\mathrm{H} \alpha$ show a second velocity component redshifted by $\sim 150 \mathrm{~km} \mathrm{~s}^{-1}$ in the nuclear region. If the prominent emission in $\mathrm{H} \alpha$ and [O III] is seen from a larger velocity range, it may be noted that the most 

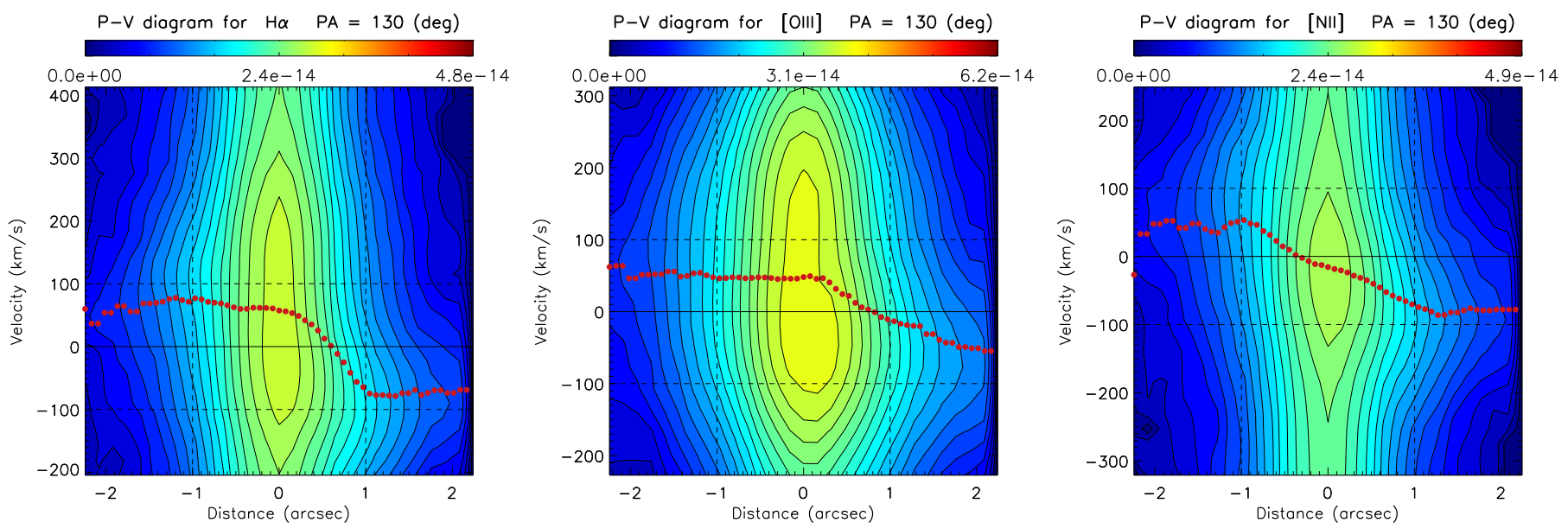

Fig. 10. From left to right: Position-velocity diagrams of $\mathrm{H} \alpha,[\mathrm{O} \mathrm{III}]$ and $[\mathrm{N} \mathrm{II}]$ emission lines orientated at $P A=130^{\circ}$, which is along the kinematic major axis (and the ionization cone). We note that the spectra are continuum subtracted. Colours and contours denote flux $\left(\mathrm{erg} \mathrm{cm}^{-2} \mathrm{~s}^{-1} \AA^{-1}\right.$ ) following the colour bar above each panel. The corresponding rotation curves obtained from the single Gaussian fit (see Sect. 3.3) are superimposed in red circles. The solid black line indicates the zero velocity $\left(V_{\mathrm{sys}}=3731 \mathrm{~km} \mathrm{~s}^{-1}\right)$, the dashed horizontal lines denotes velocities of $\pm 100 \mathrm{~km} \mathrm{~s}{ }^{-1}$, and the dashed vertical lines indicates offsets of $\pm 1^{\prime \prime}$ from the nucleus. The colour bar above each panel indicates the integrated flux. Contours are in powers of $\sqrt{2}$ to trace both the faint and strong emission structure.

of the emission occurs at velocities below $\pm 500 \mathrm{~km} \mathrm{~s}^{-1}$ for both these lines.

\subsection{Double Gaussian fit}

Given the clear evidence for a second velocity component in some of the emission lines, we fit the [O III] and $\mathrm{H} \alpha$ emission lines with a double Gaussian; these two lines were chosen as they have the highest $\mathrm{S} / \mathrm{N}$ among the double peaked lines. Given the clear velocity separation seen between the two components in the PV diagrams, we discriminated these components by their radial velocity (rather than, e.g. width), and they are henceforth referred to as the low-velocity component and highvelocity component. The line profile at each spaxel in the data cube was fit with a double Gaussian using a series of Python codes, mainly within the lmfit package ${ }^{3}$. To decide whether the observed line profile is a better fit with a single or double Gaussian, we use the corrected Akaike information criterion $\left(\mathrm{AIC}_{c}\right.$; Akaike 1974) with the additional caveats that all Gaussian amplitudes are positive. For the $\mathrm{H} \alpha$ emission line, the double Gaussian fit was performed after subtraction of the broad component (Fig. 1), which can be detected as far as $\sim 1^{\prime \prime}$ from the nucleus, even though the seeing was $\sim 0$ ! 7 . This broad component is also present in $\mathrm{H} \beta$ (Fig. 1).

The top three panels of Fig. 11 show a detailed example of the fitting process to the $\mathrm{H} \alpha$ emission line in a nuclear spaxel. These panels show the multi-component Gaussian fit to the $\mathrm{H} \alpha$ and [N II] $\lambda \lambda 6548,6583$ emission lines, before (left) and after (middle) the subtraction of the broad $\mathrm{H} \alpha$ component, and the subsequent double Gaussian fit to the narrow $\mathrm{H} \alpha$ emission. In the bottom panels of the same figure, we present examples of single/double (as decided by the $\mathrm{AIC}_{c}$ ) Gaussian fits for [O III] in different spaxels $((x, y)$ axes) of our data cube.

To estimate the errors in the velocities of the narrow components of $\mathrm{H} \alpha$ produced by an erroneous broad component subtraction, we use the following iterative process. For each spaxel, we vary the central velocity of the broad line over the range \pm 3 times the $1 \sigma$ velocity error reported by $\operatorname{lm} f i t$. The

https://lmfit.github.io/lmfit-py/intro.html double narrow components are then fit after subtraction of this broad line, and the results compared to those of the best fit (Fig. 12, bottom panels). We find that the narrow component velocities vary by less than $1.8 \mathrm{~km} \mathrm{~s}^{-1}$ for the low-velocity component and typically less than $6 \mathrm{~km} \mathrm{~s}^{-1}$ for the high-velocity component (in this latter two spaxels show velocity differences of up to $38 \mathrm{~km} \mathrm{~s}^{-1}$ ). The errors recorded by lmfit for the narrow $\mathrm{H} \alpha$ component velocities are negligible. Less than $3 \%$ of the spaxels which were originally better fitted with a double (instead of single) Gaussian are occasionally better fitted with a single Gaussian during our iterative process. Thus, overall, the two narrow component velocities are robust w.r.t. the seeing-smeared contamination of the (unresolved) BLR.

Does the velocity profile of the unresolved (but seeingsmeared) nuclear NLR also affect the velocities of the twocomponent Gaussian fit? It is difficult to quantify this effect, but we note that the PV diagrams of [O III] and $\mathrm{H} \alpha$ (see Figs. 10 and 12 for those along the major axis) clearly show that the velocity profiles are not symmetric about the nucleus even in the nuclear seeing disk. The velocities from the double component Gaussian fit appear to relatively well trace features seen in the PV diagrams, so any unresolved nuclear component would smooth out but not hide variations. We note however that to the NW of the nucleus, the two-component fit to the $\mathrm{H} \alpha$ line does not appear to follow the PV diagram. Instead the PV diagram is more consistent with a single Gaussian at a slightly larger redshift.

The results of the two-component fits to [O III] and $\mathrm{H} \alpha$ are shown in Fig. 12. The left four panels show the velocity fields of the two components of $\mathrm{H} \alpha$ and [O III], and the two rightmost panels of Fig. 12 show the same major axis PV diagrams as Fig. 10 (along PA $130^{\circ}$ ). But this time we overlay the velocities of each of the two velocity components (high-velocity component in red and low-velocity component in blue) along with the velocities obtained from the single Gaussian fit to the respective line (green), the velocities obtained by a single Gaussian fit to the [N II] line (brown) and the Bertola model fit to the stellar kinematics (black). According to the $\mathrm{AIC}_{c}$, the two-component fit is required only to the $\mathrm{SE}$. To the $\mathrm{NW}$, a single component fit gives better results. The lower [O III] velocity component has 

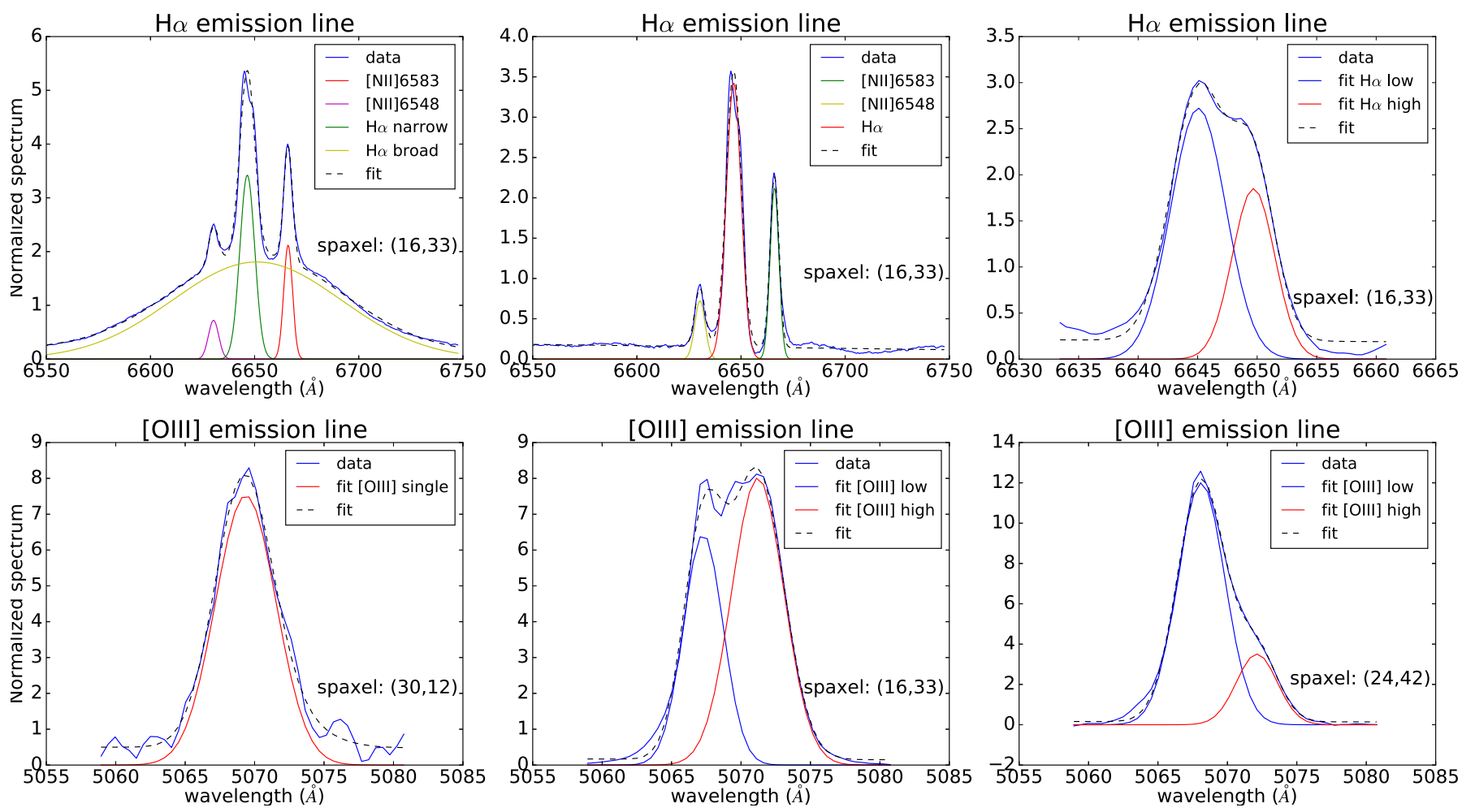

Fig. 11. Example multiple Gaussian fits to the $\mathrm{H} \alpha+[\mathrm{N}$ II] $\lambda \lambda 6548,6583 \AA$ (top panels) and [O III] $\lambda 5007 \AA$ (bottom panels) normalized emission profiles (see Sect. 3.5) for three of the six spaxels shown in Fig. 2. In the top panels we show the fitting process used for the H $\alpha+[\mathrm{N}$ II] $\lambda \lambda 6548$, $6583 \AA$ in a single nuclear spaxel: from left to right, we show: (1) the multiple Gaussian fit to $\mathrm{H} \alpha$ (one broad and one narrow component) and [N II] $\lambda \lambda 6548,6583$ (one narrow component each) emission lines, (2) the same as (1) but with the broad H $\alpha$ component subtracted, and (3) the subsequent double Gaussian fit to the $\mathrm{H} \alpha$ line. In the bottom panels, we present examples of Gaussian fits to the [O III] emission line in three different spaxels; the $\mathrm{AIC}_{c}$ is used to choose between a single or double Gaussian fit. In spaxel 30,12 (to the N) a single Gaussian provides a better fit, while in the spaxels near to the nucleus $(16,33)$ and to the $\mathrm{E}(24,42)$ double Gaussians are required.

velocities ranging from $-30 \mathrm{~km} \mathrm{~s}^{-1}$ to $-135 \mathrm{~km} \mathrm{~s}^{-1}$ and a kinematic PA of $\sim 123^{\circ}$, while the higher [O III] velocity component shows values $\approx 200 \mathrm{~km} \mathrm{~s}^{-1}$ higher. The lower $\mathrm{H} \alpha$ velocity component shows velocities of $-80 \mathrm{~km} \mathrm{~s}^{-1}$ to $70 \mathrm{~km} \mathrm{~s}^{-1}$, with a PA of $\sim 123^{\circ}$, while the higher $\mathrm{H} \alpha$ velocity component show values of $100 \mathrm{~km} \mathrm{~s}^{-1}$ up to $255 \mathrm{~km} \mathrm{~s}^{-1}$.

The corresponding velocity dispersions are shown in Fig. 13. The low-velocity component of $\mathrm{H} \alpha$ shows a centrally peaked velocity dispersion map, while in the higher velocity component shows high dispersions only in disjoint regions $\gtrsim 0$.' 6 from the nucleus. The low-velocity component of [O III] has systematically higher dispersions (except in the nucleus) in comparison to the high-velocity component.

\subsection{Black hole mass}

Black hole mass estimations for ESO 362-G18 were rigorously explored by Agís-González et al. (2014); our new observations allow us a better constraint on the value of the FWHM of $H \beta$ since we have a better spectral resolution and two-dimensional data for this line. We fit the $H \beta$ profile with a double Gaussian using the Imfit package as described in Sect. 3.5, and use the FWHM of the broad component (hereafter $\mathrm{FWHM}_{H \beta}$ ) to estimate the black hole mass.

Assuming a disk-like BLR geometry to avoid assuming a virial coefficient $f$, which can vary widely, Agís-González et al. (2014) used the following expression:

$M_{\mathrm{BH}}=R_{\mathrm{BLR}} \mathrm{FWHM}_{H \beta}^{2}\left(4 G \sin ^{2} i\right)^{-1}$ where $i$ is the angle between the LOS and the angular momentum vector of the disk-like BLR $\left(53^{\circ} \pm 5^{\circ}\right), \mathrm{R}_{\mathrm{BLR}}$ is the radius of the $\operatorname{BLR}\left(\sim 5.2 \times 10^{16} \mathrm{~cm}\right)$, and $G$ is the gravitational constant. Using our new value of $\mathrm{FWHM}_{H \beta}, 5689_{-723}^{+398} \mathrm{~km} \mathrm{~s}^{-1}$, calculated in the inner $\sim 0$ !'35 around the continuum peak (i.e. within the seeing disk), we obtain a black hole mass $M_{\mathrm{BH}}$ of $4.97_{-1.61}^{+1.60} \times 10^{7} M_{\odot}$, which is consistent with the value $\left(4.5 \pm 1.5 \times 10^{7} M_{\odot}\right)$ obtained by Agís-González et al. (2014), and in a range typical of both narrow line and broad line Seyfert 1 galaxies (Greene \& Ho 2005, Fig. 4).

\section{Discussion}

The structure map (rightmost panel of Fig. 5) shows spiral arms that get increasingly fainter as they approach the nucleus. Given the scale of the FOV and the asymmetries we find after subtracting two-dimensional Gaussians from the flux maps of each emission line, we interpret the unusual spiral arm structure as a result of instabilities produced by the inner Lindblad resonance, which is expected to lie in the inner $1.6 \mathrm{kpc}$ (Laine et al. 2002). We thus argue that the unusually high value of the parameter $p(>1.5)$ in the Bertola stellar model is a direct consequence of this resonance. The abundant dust seen in the structure map also supports the hypothesis of (Simões Lopes et al. 2007) that the presence of dust is a necessary condition for accretion onto the nuclear SMBH.

Previous studies of ESO 362-G18 have found an asymmetric [O III] emission-line morphology with a fan-shaped structure 

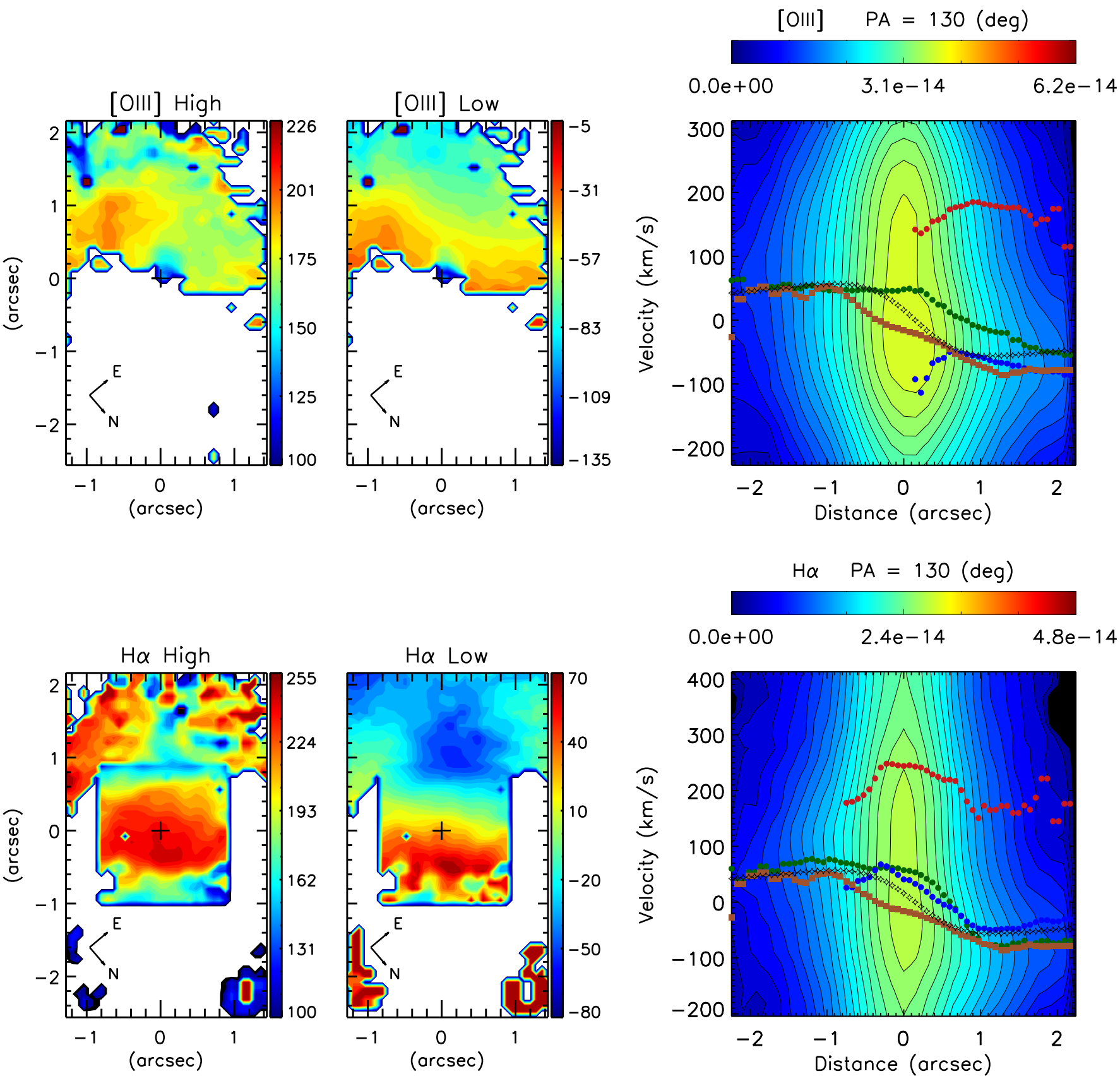

Fig. 12. Results of the double component fit to the [O III] (top) and $\mathrm{H} \alpha$ (bottom) lines. Left and middle panels: Velocity fields of the high-velocity and low-velocity narrow components, following the colour bar to the right, are shown. White denotes regions where a single Gaussian fit is preferred (see Sect. 3.5). Rightmost panels: PV diagrams as in Fig. 10, but this time we use blue and red points to denote the velocities of the low- and highvelocity components, respectively, and green points to denote the velocities obtained from the single Gaussian fitting. For comparison, we overplot the [N II] (filled brown squares) and stellar (open black stars) velocity curves along the same PA.

extending $10^{\prime \prime}$ from the nucleus to the SE, and an asymmetric morphology in the stellar continuum that is reminiscent of a minor merger system (Mulchaey et al. 1996, Fig. 29). Fraquelli et al. (2000), using long-slit spectra, noted that the kinematics of this extended emission-line region is similar to that of the stars. They posited, primarily based on the morphological appearance, the presence of an AGN outflow with a collimating axis orientated at an angle $\leq 30^{\circ}$ with respect to the galactic plane; this small angle is required to allow the nuclear radiation to intercept the gas in the disk and to allow a direct LOS to the BLR. They proposed an opening angle larger than $60^{\circ}$ for the ionizing radiation cone. We note that the spectral resolution of the longslit spectra used by Fraquelli et al. (2000) was not high enough to resolve the nuclear outflow that we posit. Our results are consistent with the outflow scenario posited by Fraquelli et al. (2000): both [N II] and [S II] share the same kinematic PA $\left(\approx 120^{\circ}\right), \approx 10^{\circ}$ less than the kinematic PA of $\mathrm{H} \alpha, \mathrm{H} \beta,\left[\mathrm{O}\right.$ I], and [O III] $\left(\approx 130^{\circ}\right)$. The offset in the kinematic centres of $\mathrm{H} \alpha, \mathrm{H} \beta$, [O I] and [O III] are closest to the direction of the ionization cone $\left(158^{\circ}\right.$, Fraquelli et al. 2000). Both facts allow us to infer that the high-ionization emission lines are more affected by this cone, while [N II] and [S II] appear to be dominantly from gas rotating in the galactic 

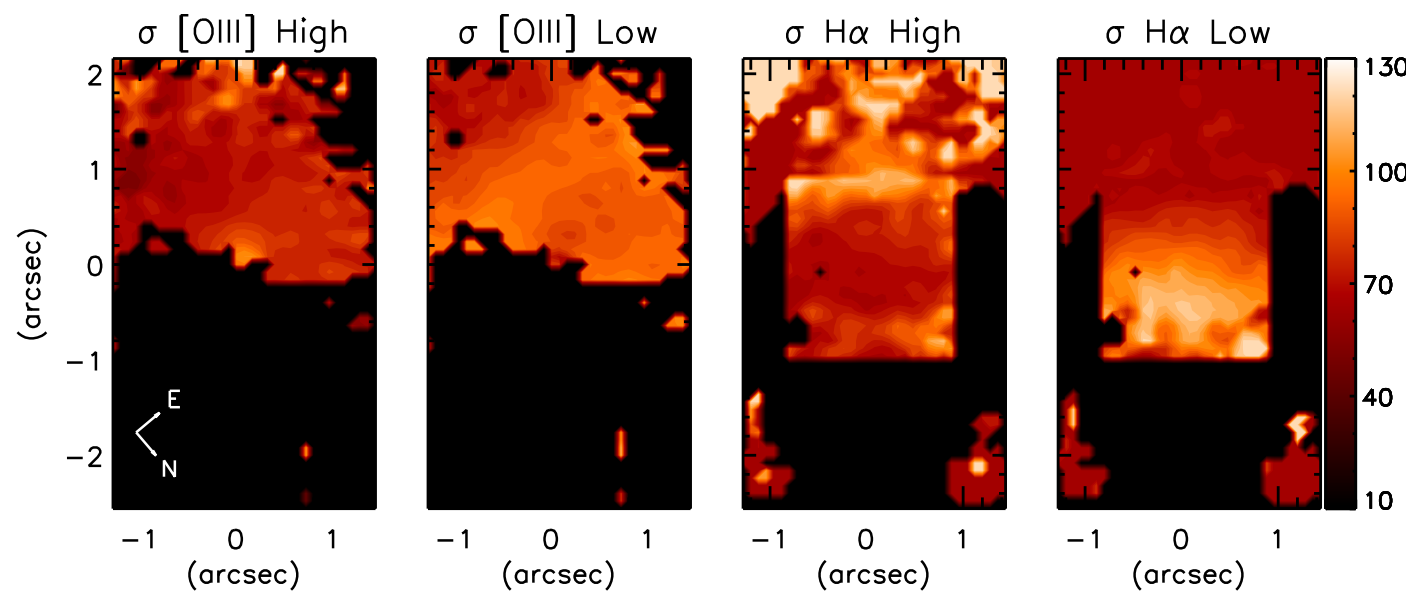

Fig. 13. From left to right: The velocity dispersion of the [O III] and $\mathrm{H} \alpha$ high-velocity and low-velocity components, respectively. All panels follow the colour bar $\left(\mathrm{km} \mathrm{s}^{-1}\right)$ shown on the right. plane, and following a rotation curve similar to that of the stars (Fig. 8).

The PV diagrams (Fig. 10) of [O III] and $\mathrm{H} \alpha$ clearly show a second velocity component $\sim 150 \mathrm{~km} \mathrm{~s}^{-1}$ to the red. Its contribution is most significant in the inner arcsecond and is the reason why the single Gaussian fit gives velocities redder than the expectation of pure rotation in the nucleus (see Fig. 7). The equivalent $\mathrm{PV}$ diagrams for $[\mathrm{O} \mathrm{I}]$ and $\mathrm{H} \beta$ (not shown) are also consistent with the presence of the second higher velocity component, but we could not rigorously fit double Gaussians to these profiles owing to their relative faintness, especially at distances $\gtrsim 1.5^{\prime \prime}$ from the nucleus (see Fig. 1). Further, both [O I] and $\mathrm{H} \beta$ (not shown) show the same asymmetries as [O III] and $\mathrm{H} \alpha$ in their velocity maps derived from a single Gaussian fit (Fig. 7).

The appearance of the velocity fields (PAs, velocity ranges, and rotation curves) of the low-velocity component of [O III] and $\mathrm{H} \alpha$ (middle panels of Fig. 12) are very similar to those derived from $[\mathrm{N} \mathrm{II}]$ in the same region. Therefore we interpret this component as emission from gas in the galactic disk that is rotating in the same manner as the [N II]-emitting gas and the stars. Only the negative velocities in [O III], reached very close to the nucleus (from $-75 \mathrm{~km} \mathrm{~s}^{-1}$ to $-120 \mathrm{~km} \mathrm{~s}^{-1}$ ), can be attributed to an outflow approaching the observer.

The high-velocity component of both these lines shows a very different velocity field, with values that exceed $200 \mathrm{~km} \mathrm{~s}^{-1}$ (>330 $\mathrm{km} \mathrm{s}^{-1}$ deprojected). We thus conclude that the highvelocity component corresponds to the bright gas within the AGN ionization cone. Given that (deprojected) velocities larger than $150 \mathrm{~km} \mathrm{~s}^{-1}$ are typically observed in outflows instead of inflows in nearby galaxies (see Barbosa et al. 2009; Storchi-Bergmann et al. 2010 and Riffel \& Storchi-Bergmann 2011 for examples of outflow velocities, and Fathi et al. 2006; Schnorr Müller et al. 2011 and Schnorr-Müller et al. 2014a for examples of inflow velocities), the most plausible explanation is that the high-velocity component is gas entrained by the AGN outflow at an angle $i$ greater than zero, located behind the plane of the sky from our LOS, and thus redshifted to the observer. Why would we preferentially see gas on the far side of the ionization cone (redshifted to us) rather than on the near side (which would be blueshifted)? The explanation lies in the illumination of the NLR clouds by the AGN: on the far side of the cone in ESO 362-G18 we see the side of the gas cloud that is illuminated by the AGN, while on the near side we see primarily the dark side of the NLR clouds (see e.g. Lena et al. 2015). The low- and high-velocity components in ESO 362-G18 are reminiscent of the case in NGC 4151 (Storchi-Bergmann et al. 2010).
The difference is that in NGC 4151 the high-velocity component corresponds to gas illuminated by a symmetric bicone, extending both in front and behind the galactic plane, while in ESO 362-G18 only the gas in front the galactic disk, illuminated by a single ionization cone, is seen.

Agís-González et al. (2014) have estimated an inner accretion disk inclination of $53^{\circ} \pm 5^{\circ}$; on the other hand, using the ratio of the minor to major photometric axis from Winkler (1997), Fraquelli et al. (2000) derived a galactic disk inclination of $\approx 37^{\circ}$. Therefore, we suggest a picture for ESO $362-\mathrm{G} 18$ in which the ionization cone has an inclination angle $i \sim 8^{\circ} \pm 5^{\circ}$ with respect to the plane of the sky with a half-opening angle of $45^{\circ}$ in such a way that the cone intersects with the galactic disk in the SE direction, illuminating gas receding from our LOS due to the outflow; this value of the half-opening angle of the ionization cone was also suggested by Agís-González et al. (2014). We only see blueshifted gas very close to the nucleus (see Fig. 12, middle top panel), where the [O III] gas is entrained by the approaching side of the cone in a small region corresponding to the thickness of the disk or bulge of the galaxy. This proposed configuration for the nuclear region in ESO 362-G18 is shown schematically in Fig. 14.

For both [O III] and $\mathrm{H} \alpha$, the highest dispersion values are predominantly reached in the low-velocity component (Fig. 13), which we interpret as gas rotation in the galactic disk. Only the central region of the high-velocity component (the outflow component) in [O III] shows dispersions higher than $105 \mathrm{~km} \mathrm{~s}^{-1}$, which we can interpret as coming from the approaching side of the outflow where the outflow is still within the galactic disk. The value of $\sigma_{H \alpha}$ of the outflow component is very sensitive to the subtraction of the broad emission in $\mathrm{H} \alpha$. This process was carried out before the two Gaussian fit, so the large dispersion values ( $\sim$ '. $8 \mathrm{SE}$ from the nucleus) should be taken with some reserve since this is potentially due to confusion with the broad emission. We can only deduce that the decrease of $\sigma_{\text {[O III] }}$ and $\sigma_{H \alpha}$ in the outflow component is due to its partial occultation by the galactic disk.

Since the outflow velocities posited above are not as high as the dividing line commonly adopted to discern immediately between starburst-driven superwinds and AGN-driven outflows (>500 $\mathrm{km} \mathrm{s}^{-1}$, Fabian 2012; Cicone et al. 2014), we must test whether the kinetic power injected by supernovae in the inner $100 \mathrm{pc}$ is sufficient to drive the outflow. The outflow, while it extends over at least $\sim 0.5 \mathrm{kpc}$ to the $\mathrm{SE}$, is already seen at high velocities in the inner seeing disk $(\sim 0.1 \mathrm{kpc})$. The global SFR of ESO 362-G18 is relatively low: the $\mathrm{H} \alpha$-derived global SFR 


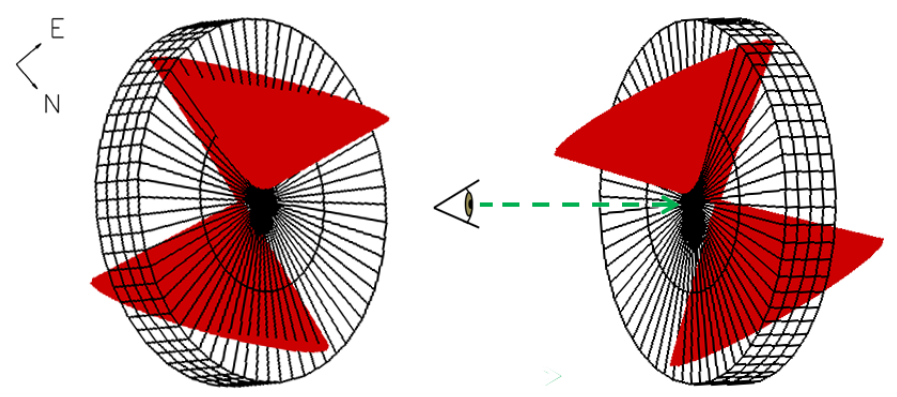

Fig. 14. Proposed configuration for the nuclear region of ESO 362-G18 (see Sect. 4) where the galactic disk is shown both from our perspective (left; in the same orientation as the data cube) and in profile (right). The SE (top) ionization cone is towards us and intersects the galaxy disk primarily behind the plane of the sky, thus generating the high-velocity (redshifted) component of [O III] and $\mathrm{H} \alpha$. This cone also intersects the galaxy disk in front of the plane of the sky, but only in a very small region close to the nucleus, producing the greatest blueshift seen in the low-velocity component of [O III]. The NW (bottom) ionization cone, if it does exist, is almost always hidden by the galactic disk for our LOS.

is $\sim 5.5 \times 10^{-3} M_{\odot} \mathrm{yr}^{-1}$ (Galbany et al. 2016) and we derive a similar value from the far-infrared luminosity (based on IRAS fluxes). We note that Meléndez et al. (2008) quoted a relatively high instantaneous SFR of $0.85 M_{\odot} \mathrm{yr}^{-1}$ in the inner kpc, based on Spitzer spectroscopy of the [Ne II] line; these authors, however, made many estimations and assumptions beyond those previously used (e.g. Genzel et al. 1998; Ho \& Keto 2007) in the disentanglement of the AGN versus star formation contribution to the [Ne II] line and its posterior conversion to a SFR method. Given that the outflow is seen in the inner kpc and starts within the inner $\sim 100 \mathrm{pc}$, the SFR in this corresponding nuclear region is expected to be significantly lower than $10^{-2} M_{\odot} \mathrm{yr}^{-1}$.

Using the relationship of Veilleux et al. (2005), $P_{\text {kin,SF }}\left(\mathrm{erg} \mathrm{s}^{-1}\right)=7 \times 10^{41} \mathrm{SFR}\left(M_{\odot} \mathrm{yr}^{-1}\right), \quad$ the galaxy-wide kinetic power injected by supernovae, $P_{\text {kin,SF }}$, is $\sim 10^{40} \mathrm{erg} \mathrm{s}^{-1}$, which is similar to the kinetic power of the outflow (see Sect. 4.2). However, the typical SFRs of galaxies with candidate starburst-driven outflows are in the range $1 M_{\odot} \mathrm{yr}^{-1}$ to $100 M_{\odot} \mathrm{yr}^{-1}$ (see e.g. Cicone et al. 2014). Attributing the outflow to the starburst thus requires a concentration of the galaxy-wide SFR in the inner $100 \mathrm{pc}$ and/or an instantaneous SFR significantly larger than the long-term average SFR and a $100 \%$ coupling of the supernova kinetic energy to the outflow. We thus conclude that starburst superwinds as the origin of the outflow in ESO 362-G18 are possible, but very unlikely.

\subsection{Feeding versus feedback}

We can estimate the mass outflow rate as the ratio of the mass of the outflowing gas to the dynamical time at the nucleus, $M_{\mathrm{g}} / t_{\mathrm{d}}$. The gas mass is given by

$M_{\mathrm{g}}=N_{\mathrm{e}} m_{\mathrm{p}} V f$,

where $N_{\mathrm{e}}$ is the electron density, $m_{\mathrm{p}}$ is the mass of the proton, $V$ is the volume of the region where the outflow is detected; we fix this value as 0.35 around the nucleus (i.e. within the seeing) and $f$ is the filling factor. The filling factor can be estimated from

$L_{H \alpha} \approx f N_{\mathrm{e}}^{2} j_{H \alpha}(T) V$,

where $j_{H \alpha}(T)=3.3534 \times 10^{-25} \mathrm{erg} \mathrm{cm}^{-3} \mathrm{~s}^{-1}$ (Osterbrock 1989) and $L_{H \alpha}$ is the $\mathrm{H} \alpha$ luminosity emitted within the volume $V$. Substituting Eq. (2) into Eq. (1) we have
$M_{g}=\frac{m_{p} L_{H \alpha}}{N_{e} j_{H \alpha}(T)}$

In Sect. 4, we concluded that the high-velocity component detected both in [O III] and $\mathrm{H} \alpha$ are produced by the emission of clouds in front of the galactic disk, which is entrained by the AGN outflow. Therefore, we estimate the $\mathrm{H} \alpha$ luminosity from the highest velocity component, yielding $L_{\alpha}=1.5 \times 10^{40} \mathrm{erg} \mathrm{s}^{-1}$, considering a luminosity distance to ESO $362-\mathrm{G} 18$ of $52.1 \mathrm{Mpc}$ (from the NASA/IPAC Extragalactic Database). On the other hand, we assumed the $N_{\mathrm{e}}$ as the mean value of $2453 \mathrm{~cm}^{-3}$ within the inner 0 .'35 from the nucleus. Taking into account all those values, we estimate an ionized gas mass of $1.5 \times 10^{4} M_{\odot}$ for the outflow component.

The dynamical time $t_{d}$ can be estimated as the ratio of the radius where we are considering the outflow $\left(0{ }^{\prime} 35 \approx 86 \mathrm{pc}\right)$ to the mean deprojected velocity of the outflow $\left(\sim 394 \mathrm{~km} \mathrm{~s}^{-1}\right)$. This gives a $t_{d}$ of $\sim 2 \times 10^{5}$ years. Finally, the mass outflow rate $\dot{M}$ is $0.074 M_{\odot} \mathrm{yr}^{-1}$. We point out that this $\dot{M}$ is only a lower limit since it corresponds only to the outflowing mass associated with the ionized side of the clouds. Additionally, if we consider a biconical outflow, assuming most of the far side of the bicone is hidden by the galactic disk, the $\dot{M}$ can be twice the calculated value, that is, $\dot{M} \approx 0.15 M_{\odot} \mathrm{yr}^{-1}$, in agreement with others $\dot{M}$ observed in nearby galaxies (Barbosa et al. 2009; Müller-Sánchez et al. 2011; Lena et al. 2015)

We can now compare the mass outflow rate with the mass accretion rate required to feed the SMBH $\dot{M}_{\text {acc }}$, which can be estimated as

$\dot{M}_{\mathrm{acc}}=\frac{L_{\mathrm{bol}}}{\eta c^{2}}$,

where $c$ is the speed of light and $L_{\text {bol }}$ is the bolometric luminosity. Using the $L_{\text {bol }}$ estimated by (Agís-González et al. 2014) of $1.3 \times 10^{44} \mathrm{erg} \mathrm{s}^{-1}$, we derive a mass accretion rate of $2.2 \times 10^{-2} M_{\odot} \mathrm{yr}^{-1}$, where we assume the radiative efficiency, $\eta$, to 0.1 , the typical value derived from Shakura-Sunyaev accretion models onto a non-rotating black hole (Shakura \& Sunyaev 1973). Therefore, the $\dot{M}_{\text {acc }}$ is $\sim 7$ times lower than the mass outflow rate. This is consistent with previous works in nearby galaxies (Barbosa et al. 2009; Müller-Sánchez et al. 2011) and indicates that most of the observed outflowing gas is mass entrained by the surrounding interstellar medium (Veilleux et al. 2005; Storchi-Bergmann 2014).

In a sample of 15 pairs of Seyfert plus inactive galaxies, Dumas et al. (2007) found that SMBHs with accretion rates larger than $10^{-4.5} M_{\odot} \mathrm{yr}^{-1}$ tend to lie in galaxies with disturbed kinematics. However, in the case of ESO 362-G18, although the value of $\dot{M}_{\text {acc }}$ is almost a thousand times greater than $10^{-4.5} M_{\odot} \mathrm{yr}^{-1}$, large twists in the gas kinematics or significant misalignments between the gas and stellar kinematics are not observed. Thus, the nuclear activity in ESO 362-G18 may be related to major mergers in the past (Hopkins et al. 2010) that do not leave current disturbances in its kinematics, rather than perturbations in the ionized gas or misalignments between the stellar and gas rotations (Dumas et al. 2007). The posited minor merger observed in the acquisition image (Fig. 1) produces no discernible disturbances in the gas or stellar kinematics within our nuclear FOV.

Considering the galactic disk as well as the BLR from the broad component of $\mathrm{Ha}$, the total ionized gas mass within $\sim 84$ pc of the nucleus is $\sim 3.3 \times 10^{5} M_{\odot}$. Assuming that this gas lies in the disk and that a small fraction $(\sim 10 \%)$ suffers a radial 
inflow within the disk, infall velocities of $\sim 34 \mathrm{~km} \mathrm{~s}^{-1}$ would be required to feed the outflow, the SMBH accretion, and maintain a SFR of $5.5 \times 10^{-3} M_{\odot} \mathrm{yr}^{-1}$ (Galbany et al. 2016) in ESO 362-G18. An inflow velocity of this magnitude (i.e. $\sim 20 \mathrm{~km} \mathrm{~s}^{-1}$ in projection if it lies in the plane of the disk) is at the limit of detectability in our observations and analysis. The residual (observed - stellar model) [N II] velocity map (rightmost panel of Fig. 6) shows blue (red) residual velocities on the far (near) side of the galaxy disk, which could be interpreted as a signature of inflow to the nucleus: the inflow velocities would then be of this order of magnitude. However, this residual pattern is primarily attributable to the mismatch between the PAs of the stellar and ionized gas kinematics (e.g. Fig. 1 of van der Kruit \& Allen 1978); the residual (observed [N II] - model [N II]) [N II] velocity map does not show this pattern. One alternative to explain the relatively high accretion rate and the relatively low inflow rate is that the AGN is now passing through a period of maximum activity, which would be transient but cause an overestimation of the accretion rate and a greater required infall velocity to maintain it. Another possibility is that the total ionized gas mass is only the tracer of the true (dominated by molecular gas) amount of available gas.

\subsection{Kinetic power}

Considering an outflow bicone, with a mass outflow rate $(\dot{M})$ of $0.148 M_{\odot} \mathrm{yr}^{-1}$, we can obtain the kinetic power $\left(\dot{E}_{\text {out }}\right)$ using the following expression

$\dot{E}_{\text {out }}=\frac{1}{2} \dot{M}\left(v^{2}+3 \sigma^{2}\right)$,

where $v$ and $\sigma$ are the average velocity and velocity dispersion of the outflowing gas, respectively. Taking these values from the nuclear region $(\leqslant 0$ !'35) of the outflow component in $\mathrm{H} \alpha$, we have $\nu=394 \mathrm{~km} \mathrm{~s}^{-1}$ and $\sigma=74 \mathrm{~km} \mathrm{~s}^{-1}$; then we obtain a kinetic power of $\dot{E}_{\text {out }}=8 \times 10^{39} \mathrm{erg} \mathrm{s}^{-1}$.

With the aim to measure the effect (feedback) of the ionized gas outflow on the galactic bulge, we compare the kinetic power with the accretion luminosity $\left(L_{\mathrm{bol}}=1.3 \times 10^{44} \mathrm{erg} \mathrm{s}^{-1}\right)$, obtaining a value of $\dot{E}_{\text {out }} / L_{\text {bol }}=6.1 \times 10^{-5}$. This is at the lower end of the range $\left(10^{-4}-5 \times 10^{-2}\right)$ found by Müller-Sánchez et al. $(2011)^{4}$. Our lower ratio could be attributed to large uncertainties in the velocity dispersion $\left(\sigma_{\text {inst }}=36 \mathrm{~km} \mathrm{~s}^{-1}\right)$ and the bolometric correction used to calculate $\mathrm{L}_{\mathrm{bol}}$ from $L_{X}(2-10 \mathrm{keV})$ in Agís-González et al. (2014). This correction can vary widely (between 4 and 110) (e.g. Lusso et al. 2012, Figs. 7 and 8).

\section{Summary and conclusions}

We observed the gaseous and stellar kinematics of the inner $0.7 \times 1.2 \mathrm{kpc}^{2}$ of the nearby Seyfert 1.5 galaxy ESO 362-G18 using optical spectra (4092-7338 $\AA$ ) from the GMOS integral field spectrograph on the Gemini South telescope, which allows the detection of a number of prominent emission lines, i.e. $\mathrm{H} \beta \lambda 4861$, [O III] $\lambda \lambda 4959,5007, \mathrm{H} \alpha+[\mathrm{N}$ II] $\lambda \lambda 6548,6583$ and [S II] $\lambda \lambda 6716,6731$. We employed a variety of IDL and Python programmes to analyze these lines and obtain spatially resolved radial velocities, velocity dispersions, and fluxes at a spatial resolution of $\sim 170 \mathrm{pc}$ and a spectral resolution of $36 \mathrm{~km} \mathrm{~s}^{-1}$. The main results of this paper are as follows.

\footnotetext{
4 With the caveat that they used the following equation for the kinetic power: $\dot{E}_{\text {out }}=\frac{1}{2} \dot{M}\left(v_{\max }^{2}+\sigma^{2}\right)$ which, for ESO $362-\mathrm{G} 18$, produces $\dot{E}_{\text {out }} / L_{\text {bol }}=8.9 \times 10^{-5}$.
}

- The $\mathrm{H} \alpha$ and [O III] lines clearly show double-peaked emission lines near to the nucleus and to the SE. We used a two Gaussian fit to separate these profiles into two kinematic components: a low-velocity component and high-velocity component.

- The stars, [N II] and [S II] emission lines, and low-velocity component of $\mathrm{H} \alpha$ and [O III] lines typically have radial velocities between $-80 \mathrm{~km} \mathrm{~s}^{-1}$ and $70 \mathrm{~km} \mathrm{~s}^{-1}$, and have very similar rotation patterns, so we interpret all of these to originate in the rotating galactic disk.

- The high-velocity component of $\mathrm{H} \alpha$ and [O III] reach values in excess of $200 \mathrm{~km} \mathrm{~s}^{-1}$ with respect to the systemic velocity, and we argue that these spectral components originate from gas outflowing within the AGN radiation cone. We present a toy model to explain why this gas is preferentially redshifted to our LOS, except at the nucleus where blueshifted [O III] emission from the outflow traces the region where the outflow is still breaking out of the galactic disk. The effects of AGN ionization has been previously observed, showing a fan-shaped morphology with an extension of $\approx 10^{\prime \prime}$ to the SE in emission-line and excitation maps.

- The assumption that the outflow component is behind the plane of the sky is also motivated by the velocity dispersions observed in [O III]: while the disk component presents the highest dispersions in most of our FOV, the outflow component exceeds it in the nuclear region, where the highest blueshift velocities are reached. This difference is consistent with attenuation from the galactic disk except very close to the nucleus, where the approaching side of the cone can be seen.

- The structure of the nuclear region of ESO 362-G18 presents spiral arms in a trailing pattern, which are increasingly fainter as we approach the nucleus. Considering the linear scale of our observations, we posit that the unusual dust morphology is a result of instabilities produced near to the inner Lindblad resonance, which is expected within the inner $1.6 \mathrm{kpc}$. The presence of the dust structures also supports the hypothesis of (Simões Lopes et al. 2007) that the presence of dust is a necessary condition for the nuclear activity in AGNs.

- While morphologically there is evidence that ESO 362-G18 is participating in a minor merger, we do not find any effect of this in the stellar or gas kinematics within our relatively small FOV.

- Using the $\mathrm{H} \alpha$ luminosity, we estimate a lower limit for the mass outflow rate $\dot{M}$ of $0.074 M_{\odot} \mathrm{yr}^{-1}$. This value will double if a biconical outflow is assumed. Further, the value we calculate is likely a lower limit to the outflow mass and rate, as we have argued that our $\mathrm{H}_{\alpha}$ luminosity used to calculate the outflow gas mass only represents the fraction of the NLR gas clouds illuminated by the AGN, rather than all of the outflowing NLR gas. In any case, our estimated outflow rate is significantly higher than the accretion rate necessary to sustain the AGN bolometric luminosity, that is, $\dot{M}_{\text {acc }} \sim 2.2 \times 10^{-2} M_{\odot} \mathrm{yr}^{-1}$.

Acknowledgements. This work is based on observations obtained at the Gemini Observatory, which is operated by the Association of Universities for Research in Astronomy, Inc., under a cooperative agreement with the NSF on behalf of the Gemini partnership: the National Science Foundation (United States), the Science and Technology Facilities Council (United Kingdom), the National Research Council (Canada), CONICYT (Chile), the Australian Research Council (Australia), Ministerio da Ciência e Tecnologia (Brazil) and south-east CYT (Argentina). NN gratefully acknowledges support from the Chilean BASAL Centro de Excelencia en Astrofísica y Tecnologías Afines (CATA) grant PFB-06/2007. PH, NN, PS and DM acknowledge support from Fondecyt 
1171506. VF acknowledges support from CONICYT Astronomy Program-2015 Research Fellow GEMINI-CONICYT (32RF0002). R.A.R. acknowledges support from FAPERGS (project No. 16/2551-0000251-7) and CNPq (project N0. $303373 / 2016-4)$. This research has made use of the NASA/IPAC Extragalactic Database (NED) which is operated by the Jet Propulsion Laboratory, California Institute of Technology, under contract with the National Aeronautics and Space Administration.

\section{References}

Agís-González, B., Miniutti, G., Kara, E., et al. 2014, MNRAS, 443, 2862 Akaike, H. 1974, IEEE Trans. Autom. Control, 19, 716

Barbosa, F. K. B., Storchi-Bergmann, T., Cid Fernandes, R., Winge, C., \& Schmitt, H. 2009, MNRAS, 396, 2

Begelman, M. C., Blandford, R. D., \& Rees, M. J. 1984, Rev. Mod. Phys., 56, 255

Bennert, N., Jungwiert, B., Komossa, S., Haas, M., \& Chini, R. 2006, A\&A, 459, 55

Bertola, F., Bettoni, D., Danziger, J., et al. 1991, ApJ, 373, 369

Bisnovatyi-Kogan, G. S., \& Lovelace, R. V. E. 2001, New A Rev., 45, 663

Bridle, A. H., \& Perley, R. A. 1984, ARA\&A, 22, 319

Cappellari, M., \& Emsellem, E. 2004, PASP, 116, 138

Cicone, C., Maiolino, R., Sturm, E., et al. 2014, A\&A, 562, A21

Cid Fernandes, R., Stasińska, G., Schlickmann, M. S., et al. 2010, MNRAS, 403 1036

Combes, F., García-Burillo, S., Casasola, V., et al. 2014, A\&A, 565, A97

Corwin, H. G. Jr., de Vaucouleurs, A., de Vaucouleurs, G. 1985, Southern Galaxy Catalogue, Univ. Texas Monogr. Astron., 4, 1

Crenshaw, D. M., Kraemer, S. B., \& Gabel, J. R. 2003, AJ, 126, 1690

Davies, R. I., Maciejewski, W., Hicks, E. K. S., et al. 2014, ApJ, 792, 101

de Vaucouleurs, G., de Vaucouleurs, A., Corwin, Jr. H. G., et al. 1991, Third Reference Catalogue of Bright Galaxies, Volumes I, II, III (New York: Springer)

Di Matteo, T., Springel, V., \& Hernquist, L. 2005, Nature, 433, 604

Dumas, G., Mundell, C. G., Emsellem, E., \& Nagar, N. M. 2007, MNRAS, 379, 1249

Emsellem, E., Fathi, K., Wozniak, H., et al. 2006, MNRAS, 365, 367

Englmaier, P., \& Shlosman, I. 2004, ApJ, 617, L115

Erwin, P., \& Sparke, L. S. 1999, in Galaxy Dynamics - A Rutgers Symposium, eds. D. R. Merritt, M. Valluri, \& J. A. Sellwood, ASP Conf. Ser., 182, 243

Fabian, A. C. 2012, ARA\&A, 50, 455

Fathi, K., Storchi-Bergmann, T., Riffel, R. A., et al. 2006, ApJ, 641, L25

Fischer, T. C., Crenshaw, D. M., Kraemer, S. B., \& Schmitt, H. R. 2013, ApJS, 209,1

Fraquelli, H. A., Storchi-Bergmann, T., \& Binette, L. 2000, ApJ, 532, 867

Galbany, L., Anderson, J. P., Rosales-Ortega, F. F., et al. 2016, MNRAS, 455, 4087

Genzel, R., Lutz, D., Sturm, E., et al. 1998, ApJ, 498, 579

Gimeno, G., Roth, K., Chiboucas, K., et al. 2016, in Ground-based and Airborne Instrumentation for Astronomy VI, Proc. SPIE, 9908, 99082S

Greene, J. E., \& Ho, L. C. 2005, ApJ, 627, 721

Greene, J. E., Zakamska, N. L., \& Smith, P. S. 2012, ApJ, 746, 86

Gültekin, K., Richstone, D. O., Gebhardt, K., et al. 2009, ApJ, 698, 198

Hernquist, L. 1989, Nature, 340, 687

Ho, L. C., \& Keto, E. 2007, ApJ, 658, 314

Hopkins, P. F., \& Quataert, E. 2010, MNRAS, 407, 1529

Hopkins, P. F., Bundy, K., Croton, D., et al. 2010, ApJ, 715, 202

Karouzos, M., Im, M., Trichas, M., et al. 2014, ApJ, 784, 137

Knapen, J. H. 2005, Ap\&SS, 295, 85

Knapen, J. H., Shlosman, I., Heller, C. H., et al. 2000, ApJ, 528, 219

Krajnović, D., Cappellari, M., de Zeeuw, P. T., \& Copin, Y. 2006, MNRAS, 366, 787
Laine, S., Shlosman, I., Knapen, J. H., \& Peletier, R. F. 2002, ApJ, 567, 97

Laine, S., van der Marel, R. P., Rossa, J., et al. 2003, AJ, 126, 2717

Laurikainen, E., Salo, H., \& Buta, R. 2004, ApJ, 607, 103

Lena, D. 2014, ArXiv e-prints [arXiv:1409.8264]

Lena, D., Robinson, A., Storchi-Bergman, T., et al. 2015, ApJ, 806, 84

Lin, M.-Y., Davies, R. I., Burtscher, L., et al. 2016, MNRAS, 458, 1375

Lusso, E., Comastri, A., Simmons, B. D., et al. 2012, MNRAS, 425, 623

Lynden-Bell, D. 1969, Nature, 223, 690

Maciejewski, W. 2004a, MNRAS, 354, 883

Maciejewski, W. 2004b, MNRAS, 354, 892

Makarov, D., Prugniel, P., Terekhova, N., Courtois, H., \& Vauglin, I. 2014, A\&A, 570, A 13

Malkan, M. A., Gorjian, V., \& Tam, R. 1998, ApJS, 117, 25

Martini, P., Regan, M. W., Mulchaey, J. S., \& Pogge, R. W. 2003, ApJ, 589, 774

Meléndez, M., Kraemer, S. B., Schmitt, H. R., et al. 2008, ApJ, 689, 95

Morganti, R., Holt, J., Saripalli, L., Oosterloo, T. A., \& Tadhunter, C. N. 2007, A\&A, 476, 735

Mulchaey, J. S., Wilson, A. S., \& Tsvetanov, Z. 1996, ApJS, 102, 309

Müller-Sánchez, F., Prieto, M. A., Hicks, E. K. S., et al. 2011, ApJ, 739, 69

Nagar, N. M., Wilson, A. S., Mulchaey, J. S., \& Gallimore, J. F. 1999, ApJS, 120, 209

Nesvadba, N. P. H., Polletta, M., Lehnert, M. D., et al. 2011, MNRAS, 415, 2359

Osterbrock, D. E. 1989, Astrophysics of gaseous nebulae and active galactic nuclei (Mill Valley: University Science Books)

Osterbrock, D. E., \& Ferland, G. J. 2006, Astrophysics of gaseous nebulae and active galactic nuclei, 2nd. ed. (California: University Science Books)

Paturel, G., Theureau, G., Bottinelli, L., et al. 2003, A\&A, 412, 57

Pogge, R. W., \& Martini, P. 2002, ApJ, 569, 624

Riffel, R. A. 2010, Ap\&SS, 327, 239

Riffel, R. A., \& Storchi-Bergmann, T. 2011, MNRAS, 411, 469

Riffel, R., Rodríguez-Ardila, A., \& Pastoriza, M. G. 2006, A\&A, 457, 61

Rodríguez-Ardila, A., Pastoriza, M. G., \& Donzelli, C. J. 2000, ApJS, 126, 63

Rupke, D. S. N., \& Veilleux, S. 2011, ApJ, 729, L27

Sánchez-Blázquez, P., Peletier, R. F., Jiménez-Vicente, J., et al. 2006, MNRAS, 371,703

Sarzi, M., Falcón-Barroso, J., Davies, R. L., et al. 2006, MNRAS, 366, 1151

Schnorr Müller, A., Storchi-Bergmann, T., Riffel, R. A., et al. 2011, MNRAS, 413, 149

Schnorr-Müller, A., Storchi-Bergmann, T., Nagar, N. M., \& Ferrari, F. 2014a, MNRAS, 438, 3322

Schnorr-Müller, A., Storchi-Bergmann, T., Nagar, N. M., et al. 2014b, MNRAS, 437, 1708

Schnorr-Müller, A., Storchi-Bergmann, T., Ferrari, F., \& Nagar, N. M. 2017, MNRAS, 466, 4370

Shakura, N. I., \& Sunyaev, R. A. 1973, in X- and Gamma-Ray Astronomy, ed. H. Bradt \& R. Giacconi, IAU Symp., 55, 155

Shlosman, I., Begelman, M. C., \& Frank, J. 1990, Nature, 345, 679

Simões Lopes, R. D., Storchi-Bergmann, T., de Fátima Saraiva, M., \& Martini, P. 2007, ApJ, 655, 718

Storchi-Bergmann, T. 2014, in Resolving The Future Of Astronomy With LongBaseline Interferometry, eds. M. J. Creech-Eakman, J. A. Guzik, \& R. E. Stencel, ASP Conf. Ser., 487, 199

Storchi-Bergmann, T., Lopes, R. D. S., McGregor, P. J., et al. 2010, MNRAS, 402, 819

Sturm, E., González-Alfonso, E., Veilleux, S., et al. 2011, ApJ, 733, L16

Treister, E., Schawinski, K., Urry, C. M., \& Simmons, B. D. 2012, ApJ, 758, L39

Tsvetanov, Z. I., \& Petrosian, A. R. 1995, ApJS, 101, 287

Valdes, F., Gupta, R., Rose, J. A., Singh, H. P., \& Bell, D. J. 2004, ApJS, 152, 251

van der Kruit, P. C., \& Allen, R. J. 1978, ARA\&A, 16, 103

Veilleux, S., Cecil, G., \& Bland-Hawthorn, J. 2005, ARA\&A, 43, 769

Winkler, H. 1997, MNRAS, 292, 273 